\title{
Boundary action of free AdS higher-spin gauge fields and the holographic correspondence
}

\author{
Euihun Joung ${ }^{a}$ and Jihad Mourad ${ }^{b}$ \\ ${ }^{a}$ Scuola Normale Superiore and INFN, \\ Piazza dei Cavalieri 7, 56126 Pisa, Italy \\ ${ }^{b}$ APC, ${ }^{1}$ Université Paris VII, \\ Bâtiment Condorcet, 75205 Paris Cedex 13, France \\ E-mail: euihun.joung@sns.it, mourad@apc.univ-paris7.fr
}

ABSTRACT: We determine the boundary terms of the free higher-spin action which reproduce the AdS Fronsdal equations in an AdS manifold with a finite distance boundary. The boundary terms are further constrained by the gauge invariance of the total action. We show that, for spins larger than two, no local boundary term can restore the full gauge symmetry, and the broken symmetry corresponds to higher-spin Weyl transformations on the boundary CFT. The boundary action is used for the evaluation of the on-shell higher-spin AdS action in terms of the boundary data given by a conformal higher-spin field.

KEYwords: AdS-CFT Correspondence, Gauge Symmetry, Anomalies in Field and String Theories

ARXIV EPRINT: 1112.5620

\footnotetext{
${ }^{1}$ Unité Mixte de Recherche 7164 du CNRS
} 


\section{Contents}

1 Introduction $\quad 1$

2 Boundary action $\quad 3$

2.1 Bulk action and notations 3

2.2 Radial decomposition 4

2.3 Construction of boundary action 5

$\begin{array}{ll}2.4 & \text { Spin } 2 \text { case }\end{array}$

$\begin{array}{lll}2.5 & \text { General case } & 10\end{array}$

3 General boundary actions and anomalies $\quad 13$

3.1 Gauge-invariant boundary terms and their transformations 14

$\begin{array}{lll}3.2 & \text { Anomaly } & 15\end{array}$

4 On-shell action $\quad 17$

4.1 Symmetries and anomalies of the on-shell action 19

$\begin{array}{lll}4.2 & \text { Spin } 2 \text { case } 20\end{array}$

$\begin{array}{lll}4.3 & \text { General case } & 22\end{array}$

5 Conclusion $\quad 24$

$\begin{array}{ll}\text { A Linearized AdS Gravity } & 26\end{array}$

$\begin{array}{ll}\text { B Radial decomposition of the Fronsdal operator } & 27\end{array}$

$\begin{array}{ll}\text { C Useful identities } & 29\end{array}$

D Gauge variation of the bulk action and the boundary action $\quad 30$

$\begin{array}{ll}\text { E Counterterms } & 30\end{array}$

$\begin{array}{lll}\text { F } & \text { Traceless and transverse projection } & 31\end{array}$

\section{Introduction}

One of the most remarkable holographic correspondences is the one relating an interacting higher-spin (HS) theory ${ }^{1}$ in Anti de Sitter spacetime (AdS) to a boundary free scalar theory [18-25]. This conjectured correspondence allows, in principle, to connect the observables of the HS theory to observables in the free CFT coupled to external HS sources [26].

\footnotetext{
${ }^{1}$ For recent reviews on higher-spin theory, see e.g. the proceeding [1] (which includes the contributions [26]) and [7-10]. For recent works on massless higher-spin fields from string theory, see e.g. [11-17].
} 
Since the CFT is free, one expects to deduce many features of the HS theory and to gain a better understanding of the Vasiliev system (see e.g. [27, 28] for some reviews), the candidate of the AdS theory. Despite many checks [20, 29-41] (see also [42, 43] for the $\mathrm{AdS}_{3} / \mathrm{CFT}_{2}$ case), the state of affairs is still, in our opinion, unsatisfactory because a systematic comparison is lacking.

In this article we propose to initiate a systematic study starting from the reasonable assumption that the HS theory can be described as a series in the coupling constant with the first term being the free Fronsdal action [44]. The subject of this paper is the determination of the free on-shell Fronsdal action as a functional of the boundary data. This is the object to be compared with the quadratic part of the CFT effective action.

The AdS/CFT correspondence applied to gauge fields in the semi-classical regime states that the finite part of on-shell AdS action coincides with the generating functional for connected correlation functions of conserved currents on the boundary. These two quantities are to be matched after the identification of the asymptotic behavior of the bulk gauge fields with the source fields of the conserved currents. In the case of the HS holography, the boundary behaviors of an infinite number of HS gauge fields in AdS turn into the sources of an infinite tower of conserved currents of higher ranks. These currents, for free scalar fields, are bilinear so that the generating functional can be viewed as the finite part of the quantum effective action of the scalar field in an external HS background [45].

The ultra-violet divergences of the effective action can be handled with a cut-off and holographically they become infra-red divergences regularized by displacing the boundary of AdS to a finite distance. As a consequence, the AdS Fronsdal action needs to be supplemented by boundary terms. In the presence of a boundary, the variation of the AdS Fronsdal action leads to boundary terms whose cancellation determines the HS generalization of the spin two York-Gibbons-Hawking terms [46, 47]. This is the subject of section 2. The boundary terms have some arbitrariness because the addition of boundary terms independent of the radial derivative does not change the equations of motions. In section 3 , we constrain these terms in order to keep as much as possible of the gauge symmetries of the action. We show that it is not possible to restore all the bulk gauge symmetries with local counterterms, and identify the broken part of the symmetries. The latter generalizes to HS the radial diffeomorphism of Gravity. This broken gauge symmetry lead in general to the corresponding anomaly of the AdS on-shell action, and should match with the HS Weyl anomaly of CFT $[36,45] .{ }^{2}$ We then turn to the evaluation of the on-shell action as a functional of the boundary behavior of spin $s$ Fronsdal field. The latter actually contains more independent components than needed to couple to a rank $s$ conserved current. But we show that these components are related on-shell to each others and reduce to a $d$-dimensional spin $s$ field. This field, which we call a HS conformal field, is not subject to trace constraints, but as the classical CFT action suggests, has a HS generalization of gauge and Weyl symmetries. ${ }^{3}$ The HS Weyl symmetry of the boundary theory is in general broken by quantum corrections. The holographic dual of this is developed in sec-

\footnotetext{
${ }^{2}$ See [48] for the Gravity case.

${ }^{3}$ The conformal HS fields were considered in [23, 49-51].
} 
tion 4 where we deduce the expression of the on-shell action in terms of the boundary data provided by the $d$-dimensional conformal spin $s$ field. For spin two, we show how one can obtain the same answer as the more conventional boundary field given by the bulk field in radial gauge evaluated on the boundary. In the general case, we give the expression of the finite part of the on-shell action and show that it is identical, up to an overall constant, to the finite part of the effective action as determined in [45]. This is due to the cancellation of the bulk anomaly by the local counterterm identified in section 3. Finally, we collect our conclusions in section 5 where, we discuss our results in the light of the AdS/CFT correspondence and in particular, we compare the on shell action with the quadratic part of the CFT effective action confirming the HS holography conjecture at this level. The technical details used in the text can be found in the appendices.

\section{Boundary action}

\subsection{Bulk action and notations}

We first recall the AdS Fronsdal HS equations and action in order to explicit our notations. We adopt the Poincaré coordinate system where the $(d+1)$-dimensional AdS is parametrized by $x^{M} \in \mathbb{R}^{d} \times \mathbb{R}_{>0}$. The AdS background metric $\bar{g}_{M N}$ is given by

$$
\begin{aligned}
d s^{2}=\bar{g}_{M N} d x^{M} d x^{N} & =\frac{1}{\left(x^{d}\right)^{2}} \eta_{M N} d x^{M} d x^{N} & & {[M, N=0,1, \cdots, d] } \\
& =\frac{1}{z^{2}}\left(\eta_{\mu \nu} d x^{\mu} d x^{\nu}+d z^{2}\right) & & {[\mu, \nu=0,1, \cdots, d-1] }
\end{aligned}
$$

where $z:=x^{d}$. The constant- $z$ hyperplanes are $d$-dimensional Minkowski spaces, and the $z=0$ surface is the boundary of AdS. The regularization of the on-shell action is performed by transporting the boundary to $z_{B} \neq 0$.

It is convenient, in order to simplify the notations, to handle the tensor fields by contracting them with auxiliary variables. More precisely in the spin $s$ case, we contract the symmetric tensor $\varphi_{M_{1} \ldots M_{s}}$ with auxiliary variables $U^{A} \in \mathbb{R}^{d+1}$, by making use of the vielbein of the AdS metric: $\bar{e}_{A}^{M}(x, z)=z \delta_{A}^{M}$, as

$$
\varphi(x, z ; U):=\frac{1}{s !} U^{A_{1}} \ldots U^{A_{s}} \bar{e}_{A_{1}}^{M_{1}}(x, z) \ldots \bar{e}_{A_{s}}^{M_{s}}(x, z) \varphi_{M_{1} \ldots M_{s}}(x, z) .
$$

With this notation, the doubly traceless constraint reads $\left(\partial_{U}^{2}\right)^{2} \varphi=0$ while the equations of motion (EOM) of the massless spin $s$ field $F_{M_{1} \ldots M_{s}} \approx 0$ can be expressed as

$$
\frac{1}{s !} U^{A_{1}} \ldots U^{A_{s}} \bar{e}_{A_{1}}^{M_{1}} \ldots \bar{e}_{A_{s}}^{M_{s}} F_{M_{1} \ldots M_{s}}=\mathcal{F} \varphi(U),
$$

where the Fronsdal operator $\mathcal{F}$ acts on the auxiliary variables as well as the spacetime variables with the AdS covariant expression:

$$
\mathcal{F}=\square_{\mathrm{AdS}}-(U \cdot \mathcal{D})\left(\partial_{U} \cdot \mathcal{D}\right)+\frac{1}{2}(U \cdot \mathcal{D})^{2} \partial_{U}^{2}-U^{2} \partial_{U}^{2}-m_{s}^{2},
$$


where $\mathcal{D}$ and $\square_{\text {AdS }}$ are respectively the AdS covariant derivative and the D'Alembertian operator in auxiliary variables:

$$
\begin{aligned}
\mathcal{D}_{A} & =\bar{e}_{A}{ }^{M} \partial_{x^{M}}+\frac{1}{2} \bar{\omega}_{A B}{ }^{C} U^{B} \partial_{U^{C}}=z \partial_{x^{A}}+U^{d} \partial_{U^{A}}-U^{A} \partial_{U^{d}}, \\
\square_{\mathrm{AdS}} & =\partial_{U^{\prime}} \cdot\left(\mathcal{D}+U^{\prime d} \partial_{U^{\prime}}-U^{\prime} \partial_{U^{\prime}}\right)\left(U^{\prime} \cdot \mathcal{D}\right),
\end{aligned}
$$

and $m_{s}^{2}$ is given by

$$
m_{s}^{2}=s^{2}+(d-5) s-2(d-2) .
$$

Using these notations for the fields and EOM, we now express the action of the free massless spin $s$ field in the region $z \geq z_{B}$ of $A d S_{d+1}$ denoted in the following by $\mathcal{M}$ :

$$
\mathcal{I}_{\mathcal{M}}=-\frac{1}{2} \int_{z_{B}}^{\infty} \frac{d z}{z^{d+1}}\left\langle\varphi \mid\left(1-\frac{1}{4} U^{2} \partial_{U}^{2}\right) \mathcal{F} \varphi\right\rangle
$$

where the bracket $\langle\cdot \mid \cdot\rangle\rangle$ is defined as

$$
\langle f \mid g\rangle:=\int d^{d} x \sum_{n=0}^{\infty} \frac{1}{n !} f_{\mu_{1} \cdots \mu_{n}}(x) g^{\mu_{1} \cdots \mu_{n}}(x) .
$$

This Fronsdal action is our starting point to construct the boundary terms which allow to derive the EOM from the variational principle and which insure that the total action is invariant under the largest subset of gauge transformations. In fact, the Fronsdal equation $\mathcal{F} \varphi \approx 0$ is invariant under the gauge transformation:

$$
\delta_{\varepsilon} \varphi(x, z ; U)=U \cdot \mathcal{D} \varepsilon(x, z ; U),
$$

where the gauge parameter $\varepsilon$ is subject to the traceless constraint:

$$
\partial_{U}^{2} \varepsilon(x, z ; U)=0
$$

The gauge invariance of the Fronsdal action in AdS is due to the Bianchi identity:

$$
\left(\partial_{U} \cdot \mathcal{D}-\frac{1}{2} U \cdot \mathcal{D} \partial_{U}^{2}\right) \mathcal{F}=0
$$

\subsection{Radial decomposition}

In order to unravel the field content from the boundary point of view, we decompose the $O(d, 1)$-tensors in terms of the boundary $O(d)$-tensors. This is conveniently done by denoting $U^{A}$ as $\left(u^{\mu}, v\right)$ and by expanding the fields $\varphi(u, v)$ and the gauge parameters $\varepsilon(u, v)$ in powers of $v$ as

$$
\begin{aligned}
& \varphi(x, z ; u, v):=\sum_{r=0}^{s} \frac{v^{r}}{r !} \phi^{(s-r)}(x, z ; u), \\
& \varepsilon(x, z ; u, v):=\sum_{r=0}^{s-1} \frac{v^{r}}{r !} \epsilon^{(s-1-r)}(x, z ; u) .
\end{aligned}
$$


The doubly tracelessness and the tracelessness constraints allow to express $\phi^{(r)}$ for $r=$ $0, \cdots, s-4$ and $\epsilon^{(r)}$ for $r=0, \cdots, s-3$ as

$$
\begin{aligned}
\phi^{(s-2 n-m)} & =(-1)^{n+1}\left[(n-1)\left(\partial_{u}^{2}\right)^{n} \phi^{(s-m)}+n\left(\partial_{u}^{2}\right)^{n-1} \phi^{(s-2-m)}\right], \\
\epsilon^{(s-1-2 n-m)} & =(-1)^{n}\left(\partial_{u}^{2}\right)^{n} \epsilon^{(s-1-m)},
\end{aligned}
$$

where $n=1, \cdots$ and $m=0$ or 1 . Thus the independent gauge fields and parameters are the unconstrained

$$
\phi^{(s)}, \quad \phi^{(s-1)}, \quad \phi^{(s-2)}, \quad \phi^{(s-3)},
$$

and

$$
\epsilon^{(s-1)}, \quad \epsilon^{(s-2)}
$$

In terms of these $O(d)$-tensors, the gauge transformation (2.9) reads

$$
\begin{aligned}
\delta \phi^{(s)} & =z(u \cdot \partial) \epsilon^{(s-1)}-u^{2} \epsilon^{(s-2)} \\
\delta \phi^{(s-1)} & =\left(z \partial_{z}+s-1+u^{2} \partial_{u}^{2}\right) \epsilon^{(s-1)}+z(u \cdot \partial) \epsilon^{(s-2)} \\
\delta \phi^{(s-2)} & =-z(u \cdot \partial) \partial_{u}^{2} \epsilon^{(s-1)}+\left[2\left(z \partial_{z}+s-2\right)+u^{2} \partial_{u}^{2}\right] \epsilon^{(s-2)} \\
\delta \phi^{(s-3)} & =-\left[3\left(z \partial_{z}+s-3\right)+u^{2} \partial_{u}^{2}\right] \partial_{u}^{2} \epsilon^{(s-1)}-z(u \cdot \partial) \partial_{u}^{2} \epsilon^{(s-2)}
\end{aligned}
$$

Notice that the transformation of $\phi^{(s)}$ is a combination of a $d$-dimensional HS gauge transformation with an unconstrained parameter and a HS Weyl transformation characterizing a conformal $d$-dimensional gauge field. From the boundary point of view at constant $z$ there are two spin $s-1$ unconstrained gauge parameters $\epsilon^{(s-1)}$ and $\partial_{z} \epsilon^{(s-1)}$ and two spin $s-2$ unconstrained gauge parameters $\epsilon^{(s-2)}$ and $\partial_{z} \epsilon^{(s-2)}$. Notice also that $\partial_{z} \epsilon^{(s-1)}$ and $\partial_{z} \epsilon^{(s-2)}$ can be seen as Stueckelberg shift fields for $\phi^{(s-1)}$ and $\phi^{(s-2)}$. The latter pair of fields can thus be set to zero by fixing $\partial_{z} \epsilon^{(s-1)}$ and $\partial_{z} \epsilon^{(s-2)} \cdot{ }^{4}$

\subsection{Construction of boundary action}

In the presence of a finite distance boundary, the bulk action $\mathcal{I}_{\mathcal{M}}(2.7)$ is not sufficient to get the EOM from the variational principle. This can be easily seen by calculating the on-shell variation of the bulk action $\delta \mathcal{I}_{\mathcal{M}}$ under $\delta \varphi$ :

$$
\delta \mathcal{I}_{\mathcal{M}} \approx-\frac{1}{2} \int_{z_{B}}^{\infty} \frac{d z}{z^{d+1}}\left\langle\varphi \mid\left(1-\frac{1}{4} U^{2} \partial_{U}^{2}\right) \mathcal{F} \delta \varphi\right\rangle,
$$

where we have used the EOM. If $\mathcal{M}$ were a manifold without boundary, then integrating by part (2.19), $\delta \mathcal{I}_{\mathcal{M}}$ could be expressed with the EOM and so would vanish on-shell. But for the present case, $\mathcal{M}$ has a boundary at $z=z_{B}$ and the integration by part results in an additional boundary term. Remember also that when varying the action we should use a proper boundary condition, either the Dirichlet condition $\delta \varphi\left(z_{B}\right)=0$ or the Neumann condition $\delta \partial_{z} \varphi\left(z_{B}\right)=0$ (or a mixed one). Following the standard version of AdS/CFT,

\footnotetext{
${ }^{4}$ In eq. (2.18) and in the following, we will use the notation $\partial=\partial /(\partial x)$ and the derivative with respect to $z$ will be explicitly spelled $\partial_{z}$.
} 
we use the Dirichlet boundary condition. By integrating (2.19) by parts and by using $\delta \varphi\left(z_{B}\right)=0$ and the EOM, we obtain $\delta \mathcal{I}_{\mathcal{M}}$ as a boundary term:

$$
\delta \mathcal{I}_{\mathcal{M}} \approx \frac{z_{B}^{-d-1}}{2}\left\langle\varphi \mid\left(1-\frac{1}{4} U^{2} \partial_{U}^{2}\right) \mathcal{F}_{z z} \partial_{z} \delta \varphi\right\rangle_{z_{B}}
$$

where $\mathcal{F}_{z z}$ is the coefficient of $\partial_{z}^{2}$ in the Fronsdal operator (2.4). Explicitly it is given by

$$
\mathcal{F}_{z z}=z^{2}\left(1-v \partial_{v}+\frac{1}{2} v^{2} \partial_{U}^{2}\right)
$$

We see that the on-shell variation $\delta \mathcal{I}_{\mathcal{M}}$ does not vanish, and this implies that the variational principle does not lead to the EOM and needs the addition of a boundary term $\mathcal{I}_{\partial \mathcal{M}}^{0}$ so that the total action satisfies

$$
\delta\left(\mathcal{I}_{\mathcal{M}}+\mathcal{I}_{\partial \mathcal{M}}^{0}\right) \approx 0 .
$$

In order that the bulk action provides the Fronsdal equation from the variational principle with $\delta \varphi\left(z_{B}\right)=0$, the boundary term $\mathcal{I}_{\partial \mathcal{M}}^{0}$ is determined by

$$
\mathcal{I}_{\partial \mathcal{M}}^{0}=-\frac{z_{B}^{-d-1}}{2}\left\langle\varphi \mid\left(1-\frac{1}{4} U^{2} \partial_{U}^{2}\right) \mathcal{F}_{z z} \partial_{z} \varphi\right\rangle_{z_{B}} .
$$

By using the radial decomposition of the field $\varphi$, we obtain the explicit expression of (2.23) in terms of $O(d)$-tensor fields as

$$
\mathcal{I}_{\partial \mathcal{M}}^{0}=\frac{z_{B}^{-d}}{2}\left[\left\langle\phi^{(s)} \mid \mathrm{P}_{\mathrm{e}}^{\prime} z \partial_{z} \phi^{(s)}\right\rangle_{z_{B}}+\left\langle\left\langle\psi^{(s-3)} \mid \mathrm{P}_{\mathrm{o}}^{\prime} z \partial_{z} \psi^{(s-3)}\right\rangle_{z_{B}}\right]\right.
$$

with

$$
\psi^{(s-3)}:=\frac{1}{2}\left(\phi^{(s-3)}+3 \partial_{u}^{2} \phi^{(s-1)}\right)
$$

and

$$
\mathrm{P}_{\mathrm{e}}^{\prime}:=\sum_{n=0}^{\infty} \frac{2 n-1}{(2 n) !}\left(u^{2}\right)^{n}\left(\partial_{u}^{2}\right)^{n}, \quad \mathrm{P}_{\mathrm{o}}^{\prime}:=\sum_{n=0}^{\infty} \frac{2 n+2}{(2 n+3) !}\left(u^{2}\right)^{n}\left(\partial_{u}^{2}\right)^{n} .
$$

Notice that the boundary term $\mathcal{I}_{\partial \mathcal{M}}^{0}$ does not depend on $\phi^{(s-2)}$, and it depends on $\phi^{(s-1)}$ and $\phi^{(s-3)}$ only through $\psi^{(s-3)}$.

The action $\mathcal{I}_{\mathcal{M}}+\mathcal{I}_{\partial \mathcal{M}}^{0}$ provides the Fronsdal equation in $\mathcal{M}$, but, since we are using a Dirichlet boundary condition, any boundary term which does not involve $\partial_{z} \phi^{(r)}$ can be freely added without affecting the variational principle. This gives rise to an ambiguity on the on-shell action and consequently in the AdS/CFT correspondence. In order to fix the ambiguity, we rely on the variation under the gauge transformation of the total action:

$$
\delta_{\varepsilon}\left(\mathcal{I}_{\mathcal{M}}+\mathcal{I}_{\partial \mathcal{M}}\right)=0
$$

where $\mathcal{I}_{\partial \mathcal{M}}$ is a completion of $\mathcal{I}_{\partial \mathcal{M}}^{0}$ with $\partial_{z} \phi^{(r)}$-free boundary terms. For the determination of $\mathcal{I}_{\partial \mathcal{M}}$, we proceed in two steps: 
1. we determine the gauge variation of $\mathcal{I}_{\mathcal{M}}+\mathcal{I}_{\partial \mathcal{M}}^{0}$ under the transformations $\alpha$ generated by $\epsilon^{(s-1)}, \partial_{z} \epsilon^{(s-1)}$ and $\partial_{z} \epsilon^{(s-2)}$, and then add a $\partial_{z} \phi^{(r)}$-independent boundary term such that

$$
\delta_{\alpha}\left(\mathcal{I}_{\mathcal{M}}+\mathcal{I}_{\partial \mathcal{M}}\right)=0
$$

The result of this step is summarised in eqs. (2.69) and (2.70).

2. The most general boundary term is then given by the addition to the above term of a $\partial_{z} \phi^{(r)}$-independent local term invariant under the above transformations $\alpha$. We determine these counterterms and examine whether they can compensate the gauge variation of $\mathcal{I}_{\mathcal{M}}+\mathcal{I}_{\partial \mathcal{M}}$ under $\epsilon^{(s-2)}$. The outcome of this step are the counterterms (3.7) and (3.8) with the definitions (3.3) and (3.10). We will also prove in section 3.2 that, for spin larger than two, no counterterm can fully compensate the $\epsilon^{(s-2)}$ variation and hence the action is characterized by an anomaly under the HS Weyl transformations.

Let us first specialize to the spin 2 case for which the formulas simplify considerably.

\section{$2.4 \quad$ Spin 2 case}

The boundary term (2.24) reduces to

$$
\mathcal{I}_{\partial \mathcal{M}}^{0}=-\frac{z_{B}^{-d}}{2}\left\langle\phi^{(2)} \mid\left(1-\frac{1}{2} u^{2} \partial_{u}^{2}\right) z \partial_{z} \phi^{(2)}\right\rangle_{z_{B}} .
$$

Any boundary term independent of $\partial_{z} \phi^{(r)}$ vanishes under the variation $\delta \varphi$ with the Dirichlet condition, and can be freely added without effecting the EOM.

In order to fix this ambiguity of boundary terms, as stated above, we will rely on the gauge invariance. The linearized diffeorphism is expressed in auxiliary variables in eq. (2.9), and in terms of $\phi^{(2)}, \phi^{(1)}, \phi^{(0)}(2.12)$ and $\epsilon^{(1)}, \epsilon^{(0)}$ (2.13), the gauge transformation (2.18) reads

$$
\begin{aligned}
& \delta \phi^{(2)}=z(u \cdot \partial) \epsilon^{(1)}-u^{2} \epsilon^{(0)} \\
& \delta \phi^{(1)}=\left(z \partial_{z}+1\right) \epsilon^{(1)}+z(u \cdot \partial) \epsilon^{(0)} \\
& \delta \phi^{(0)}=2 z \partial_{z} \epsilon^{(0)}
\end{aligned}
$$

Notice that the transformation of $\phi^{(2)}$ is a combination of a $d$-dimensional gauge transformation and a Weyl transformation characterizing a conformal $d$-dimensional spin 2 gauge field. From the boundary point of view at constant $z$ there are two spin 1 gauge parameters $\epsilon^{(1)}\left(z_{B}\right)$ and $\partial_{z} \epsilon^{(1)}\left(z_{B}\right)$ and two spin 0 gauge parameters $\epsilon^{(0)}\left(z_{B}\right)$ and $\partial_{z} \epsilon^{(0)}\left(z_{B}\right)$. Notice also that $\partial_{z} \epsilon^{(1)}\left(z_{B}\right)$ and $\partial_{z} \epsilon^{(0)}\left(z_{B}\right)$ can be seen as Stueckelberg shift fields for $\phi^{(1)}\left(z_{B}\right)$ and $\phi^{(0)}\left(z_{B}\right)$. The latter pair of fields can thus be set to zero by fixing $\partial_{z} \epsilon^{(1)}\left(z_{B}\right)$ and $\partial_{z} \epsilon^{(0)}\left(z_{B}\right)$.

Under the gauge transformation (2.30) the bulk action $\mathcal{I}_{\mathcal{M}}$ is not invariant but gives a boundary term because the gauge invariance of the linearized Gravity action requires again 
an integration by part. More precisely the gauge variation of the bulk action is given with the aid of the Bianchi identity by the following boundary term:

$$
\delta_{\varepsilon} \mathcal{I}_{\mathcal{M}}=\frac{z_{B}^{-d}}{2}\left\langle v \varepsilon \mid\left(1-\frac{1}{4} U^{2} \partial_{U}^{2}\right) \mathcal{F} \varphi\right\rangle_{z_{B}}
$$

Note that the bulk action is still invariant under the gauge transformation whose gauge parameter vanishes on the boundary: $\varepsilon\left(z_{B}\right)=0$. We want to complete $\mathcal{I}_{\partial \mathcal{M}}^{0}$ with suitable $\partial_{z} \phi^{(r)}$-free boundary terms so that the resulting boundary term $\mathcal{I}_{\partial \mathcal{M}}$ satisfies

$$
\delta_{\epsilon^{(r)}}\left(\mathcal{I}_{\mathcal{M}}+\mathcal{I}_{\partial \mathcal{M}}\right)=0 \quad[r=1,0] .
$$

To begin with, we construct a boundary term which restores the gauge invariance under $\epsilon^{(1)}$ (that is, both of $\epsilon^{(1)}\left(z_{B}\right)$ and $\partial_{z} \epsilon^{(1)}\left(z_{B}\right)$ ).

For that, we need to first compute the gauge variations of $\mathcal{I}_{\mathcal{M}}$ and $\mathcal{I}_{\partial \mathcal{M}}^{0}$ under $\epsilon^{(1)}$, and then construct a suitable boundary term to cancel them. It is in fact more convenient to consider, instead of $\mathcal{I}_{\partial \mathcal{M}}^{0}$, a complemented one $\mathcal{I}_{\partial \mathcal{M}}^{0^{\prime}}$ whose gauge variation is easier to compute. This is equivalent since in any case we should add another boundary term to compensate the gauge variation. Let us replace $z \partial_{z} \phi^{(2)}$ in the l.h.s. of $\mathcal{I}_{\partial \mathcal{M}}^{0}(2.29)$ with a $\epsilon^{(1)}\left(z_{B}\right), \partial_{z} \epsilon^{(1)}\left(z_{B}\right)$ and $\partial_{z} \epsilon^{(0)}\left(z_{B}\right)$-invariant:

$$
\chi^{(2)}:=z \partial_{z} \phi^{(2)}-z(u \cdot \partial) \phi^{(1)}+\frac{1}{2} u^{2} \phi^{(0)} .
$$

The complemented boundary term containing $\partial_{z} \phi^{(2)}$ becomes now

$$
\mathcal{I}_{\partial \mathcal{M}}^{0^{\prime}}=\frac{z_{B}^{-d}}{2}\left\langle\phi^{(2)} \mid\left(-1+\frac{1}{2} u^{2} \partial_{u}^{2}\right) \chi^{(2)}\right\rangle_{z_{B}},
$$

and its $\epsilon^{(1)}$-variation is given by

$$
\delta_{\epsilon^{(1)}} \mathcal{I}_{\partial \mathcal{M}}^{0^{\prime}}=\frac{z_{B}^{-d+1}}{2}\left\langle\epsilon^{(1)} \mid\left(\partial_{u} \cdot \partial-u \cdot \partial \partial_{u}^{2}\right) \chi^{(2)}\right\rangle_{z_{B}} .
$$

After obtaining the gauge variation of the boundary term, it is time to compute the gauge variation of the bulk action $\mathcal{I}_{\mathcal{M}}$. For the case of $\epsilon^{(1)}$-gauge variation, (2.31) reduces to

$$
\delta_{\epsilon^{(1)}} \mathcal{I}_{\mathcal{M}}=\frac{z_{B}^{-d}}{2}\left\langle\epsilon^{(1)} \mid \partial_{v} \mathcal{F} \varphi\right\rangle_{z_{B}},
$$

and we obtain $\left(\partial_{v} \mathcal{F} \varphi\right)(x, z ; u, 0)$ from a direct computation (B.3) as

$$
\begin{aligned}
\left(\partial_{v} \mathcal{F} \varphi\right)(x, z ; u, 0)= & z^{2}\left(u \cdot \partial \partial_{u}^{2}-\partial \cdot \partial_{u}\right) \partial_{z} \phi^{(2)}(x, z ; u) \\
& +z^{2}\left(\square-u \cdot \partial \partial \cdot \partial_{u}\right) \phi^{(1)}(x, z ; u)+(d-1) z u \cdot \partial \phi^{(0)}(x, z) .
\end{aligned}
$$

Finally, by summing (2.35) and (2.36), we see that the $\epsilon^{(1)}$-variation of the total action vanish:

$$
\delta_{\epsilon^{(1)}}\left(\mathcal{I}_{\mathcal{M}}+\mathcal{I}_{\partial \mathcal{M}}^{0^{\prime}}\right)=0
$$


Moreover the total action is also invariant under the gauge transformation generated by $\partial_{z} \epsilon^{(0)}\left(z_{B}\right)$ since $\phi^{(2)}$ and $\chi^{(2)}$ are invariant. In fact, the boundary term that we have just constructed corresponds to the quadratic part of the boundary term appearing in the linearized AdS Gravity, that is the Einstein-Hilbert bulk action supplemented with the York-Gibbons-Hawking term. More precisely, $\mathcal{I}_{\partial \mathcal{M}}^{0^{\prime}}$ coincides to the boundary term (A.10) of the linearized AdS Gravity (see appendix A for the details).

Let us now determine the variation of the bulk and boundary terms under the gauge transformation generated by $\epsilon^{(0)}$, the first is given by

$$
\begin{aligned}
\delta_{\epsilon^{(0)}} \mathcal{I}_{\mathcal{M}} & =\frac{z_{B}^{-d}}{4}\left\langle\left\langle\epsilon^{(0)} \mid\left(\partial_{v}{ }^{2}-\partial_{u}{ }^{2}\right) \mathcal{F} \varphi^{(2)}\right\rangle\right. \\
& =\frac{z_{B}^{-d}}{2}\left\langle\left\langle\epsilon^{(0)} \mid(d-1) \partial_{u}^{2} \chi^{(2)}-z^{2}\left[\square \partial_{u}^{2}-\left(\partial_{u} \cdot \partial\right)^{2}\right] \phi^{(2)}\right\rangle_{z_{B}},\right.
\end{aligned}
$$

and the second

$$
\delta_{\epsilon^{(0)}} \mathcal{I}_{\partial \mathcal{M}}^{0^{\prime}}=\frac{z_{B}^{-d}}{2}\left\langle\epsilon^{(0)} \mid-(d-1) \partial_{u}^{2} \chi^{(2)}-z^{2}\left[\square \partial_{u}^{2}-\left(\partial_{u} \cdot \partial\right)^{2}\right] \phi^{(2)}\right\rangle_{z_{B}} .
$$

The $\chi^{(2)}$-contribution cancels and finally we get for the total variation

$$
\delta_{\epsilon^{(0)}}\left(\mathcal{I}_{\mathcal{M}}+\mathcal{I}_{\partial \mathcal{M}}^{0^{\prime}}\right)=-z_{B}^{-d+2}\left\langle\left\langle\epsilon^{(0)} \mid\left[\square \partial_{u}^{2}-\left(\partial_{u} \cdot \partial\right)^{2}\right] \phi^{(2)}\right\rangle_{z_{B}} .\right.
$$

It is natural now to continue the procedure and look for a boundary term which is $\partial_{z} \phi^{(r)}$ independent, invariant under the $\epsilon^{(1)}, \partial_{z} \epsilon^{(1)}$ and $\partial_{z} \epsilon^{(0)}$ and which cancels the above variation. In fact, checking the gauge variations we easily see that such a term could depend only on $\phi^{(2)}$. The $\epsilon^{(1)}$-invariant terms with two derivatives reduce to

$$
\mathcal{I}_{\partial \mathcal{M}}^{\text {(c.t.) }}=c z_{B}^{-d+2}\left\langle\left\langle\phi^{(2)} \mid\left(1-\frac{1}{4} u^{2} \partial_{u}^{2}\right) \mathcal{F}_{M^{d}} \phi^{(2)}\right\rangle_{z_{B}},\right.
$$

with an undetermined constant $c$. Its variation under $\epsilon^{(0)}$ is

$$
\delta_{\epsilon^{(0)}} \mathcal{I}_{\partial \mathcal{M}}^{(\text {c.t. }}=2(d-2) c z_{B}^{-d+2} \| \epsilon^{(0)}\left|\left[\square \partial_{u}^{2}-\left(\partial_{u} \cdot \partial\right)^{2}\right] \phi^{(2)}\right\rangle_{z_{B}} .
$$

As a result, for $d \neq 2$ it is possible to cancel the term (2.41) by the addition of $\mathcal{I}_{\partial \mathcal{M}}^{\text {(c.t.) }}$ with

$$
c=\frac{1}{2(d-2)} .
$$

For $d=2$ it is not possible to cancel $(2.41)$ by a local term, this results in an anomaly under the symmetries generated by $\epsilon^{(0)}$.

In brief, we have found that in the spin 2 case the bulk action has to be amended by a boundary term in such a way that the equations of motion are correctly reproduced and that the action is invariant under all the gauge symmetries but the ones generated by $\epsilon^{(0)}$ which are anomalous for $d=2$. We now turn to the generalization of the above results to the arbitrary spin case. 


\subsection{General case}

We want to construct a boundary term $\mathcal{I}_{\partial \mathcal{M}}$ satisfying (2.28). In fact, it will be more convenient as for spin 2 to first modify $\mathcal{I}_{\partial \mathcal{M}}^{0}$ into $\mathcal{I}_{\partial \mathcal{M}}^{0^{\prime}}$ such that its $\epsilon^{(s-1)}$-variation has a simpler expression. Then, we construct a boundary term $\mathcal{I}_{\partial \mathcal{M}}^{1}$ such that

$$
\delta_{\alpha} \mathcal{I}_{\partial \mathcal{M}}^{1}=-\delta_{\alpha}\left(\mathcal{I}_{\mathcal{M}}+\mathcal{I}_{\partial \mathcal{M}}^{0^{\prime}}\right)
$$

where $\alpha$ is a gauge transformation generated by $\epsilon^{(s-1)}$ and $\partial_{z} \epsilon^{(s-1)}$. We will proceed in steps, from $\epsilon^{(s-1)}$ and $\partial_{z} \epsilon^{(s-1)}$ transformations we will construct the first boundary term $\mathcal{I}_{\partial \mathcal{M}}^{1}$ and then from the transformation of $\mathcal{I}_{\mathcal{M}}+\mathcal{I}_{\partial \mathcal{M}}^{0^{\prime}}+\mathcal{I}_{\partial \mathcal{M}}^{1}$ under $\partial_{z} \epsilon^{(s-2)}$ we determine $\mathcal{I}_{\partial \mathcal{M}}^{2}$.

The zeroth boundary term $\mathcal{I}_{\partial \mathcal{M}}^{0}(2.24)$ contains $\partial_{z} \phi^{(s)}$ and $\partial_{z} \psi^{(s-3)}$ on the r.h.s. of the brackets. They can be replaced by the invariants under the $\epsilon^{(s-1)}$-transformation by adding $\partial_{z} \phi^{(r)}$-independent terms as

$$
\begin{aligned}
\chi^{(s)} & :=\left(z \partial_{z}+s-2\right) \phi^{(s)}-z(u \cdot \partial) \phi^{(s-1)}-u^{2} \phi^{(s-2)}, \\
\chi^{(s-3)} & :=\left(z \partial_{z}+s-3\right) \psi^{(s-3)}+(d+2 s-5) \phi^{(s-3)}+\frac{1}{2} u^{2} \partial_{u}^{2}\left(\phi^{(s-3)}+\partial_{u}^{2} \phi^{(s-1)}\right),
\end{aligned}
$$

so that $\mathcal{I}_{\partial \mathcal{M}}^{0}$ is replaced by

$$
\mathcal{I}_{\partial \mathcal{M}}^{0^{\prime}}=\frac{z_{B}^{-d}}{2}\left[\left\langle\phi^{(s)} \mid \mathrm{P}_{\mathrm{e}}^{\prime} \chi^{(s)}\right\rangle_{z_{B}}+\left\langle\left\langle\psi^{(s-3)} \mid \mathrm{P}_{\mathrm{o}}^{\prime} \chi^{(s-3)}\right\rangle_{z_{B}}\right] .\right.
$$

Since $\chi^{(s)}$ and $\chi^{(s-3)}$ are $\epsilon^{(s-1)}$-invariants, the $\epsilon^{(s-1)}$-variation of the above is simple to compute, and is given by

$$
\delta_{\epsilon^{(s-1)}} \mathcal{I}_{\partial \mathcal{M}}^{0^{\prime}}=\frac{z_{B}^{-d}}{2}\left\langle\epsilon^{(s-1)} \mid \mathrm{P}_{\mathrm{e}}\left[z\left(\partial_{u} \cdot \partial-u \cdot \partial \partial_{u}^{2}\right) \chi^{(s)}+u^{2} \chi^{(s-3)}\right]\right\rangle_{z_{B}},
$$

with

$$
\mathrm{P}_{\mathrm{e}}:=\sum_{n=0}^{\infty} \frac{1}{(2 n) !}\left(u^{2}\right)^{n}\left(\partial_{u}^{2}\right)^{n}, \quad \mathrm{P}_{\mathrm{o}}:=\sum_{n=0}^{\infty} \frac{1}{(2 n+1) !}\left(u^{2}\right)^{n}\left(\partial_{u}^{2}\right)^{n}
$$

In order to get (2.48), we have used the identities (C.1, C.2). To construct the first boundary term $\mathcal{I}_{\partial \mathcal{M}}^{1}$ with (2.45), we need to compute also the gauge variation of the bulk action. From the gauge invariance of the Fronsdal equation and the Bianchi identity (2.11), we get the gauge variation of the action as (2.31). For a more explicit expression of the above, we consider the radial decomposition of the Fronsdal equation:

$$
\left(\mathcal{F} \varphi^{(s)}\right)(x, z ; u, v)=\sum_{r=0}^{s} \frac{v^{r}}{r !} f^{(s-r)}(x, z ; u),
$$

where $f^{(r)}$ are determined by $f^{(s)}, f^{(s-1)}, f^{(s-2)}$ and $f^{(s-3)}$ since the Fronsdal equation also has vanishing the double-trace:

$$
\begin{aligned}
f^{(s-2 n-m)}(x, z ; u)=(-1)^{n+1}[ & (n-1)\left(\partial_{u}^{2}\right)^{n}\left(\partial_{v}^{m} \mathcal{F} \varphi\right)(x, z ; u, 0) \\
& \left.+n\left(\partial_{u}^{2}\right)^{n-1}\left(\partial_{v}^{m+2} \mathcal{F} \varphi\right)(x, z ; u, 0)\right],
\end{aligned}
$$


where $m$ is either 0 or 1 . Using (2.50) and (2.51), the gauge variation of the bulk action is written as

$$
\delta_{\varepsilon} \mathcal{I}_{\mathcal{M}}=\frac{z_{B}^{-d}}{2}\left[\left\langle\epsilon^{(s-1)} \mid \mathrm{P}_{\mathrm{e}} \partial_{v} \mathcal{F} \varphi\right\rangle+\left\langle\left\langle\epsilon^{(s-2)} \mid \mathrm{P}_{\mathrm{o}} \frac{1}{2}\left(\partial_{v}^{2}-\partial_{u}^{2}\right) \mathcal{F} \varphi\right\rangle\right] .\right.
$$

The expressions for $\left.\partial_{v} \mathcal{F} \varphi\right|_{v=0}$ and $\left.\left(\partial_{u}^{2}-\partial_{v}^{2}\right) \mathcal{F} \varphi\right|_{v=0}$ are obtained in (B.3) and (B.4) from the explicit decomposition (B.2) of the Fronsdal operator. Noting that $\left.\partial_{v} \mathcal{F} \varphi\right|_{v=0}$ is gauge invariant as the Fronsdal equation is, we express it in terms of $\epsilon^{(s-1)}$-invariants as

$$
\left.\partial_{v} \mathcal{F} \varphi\right|_{v=0}=-z\left(\partial_{u} \cdot \partial-u \cdot \partial \partial_{u}^{2}\right) \chi^{(s)}-u^{2} \chi^{(s-3)}+z u \cdot \partial \zeta^{(s-2)},
$$

where $\zeta^{(s-2)}$ is a $\epsilon^{(s-1)}$-invariant:

$$
\zeta^{(s-2)}:=z u \cdot \partial \psi^{(s-3)}+3(d+2 s-5) \phi^{(s-2)}+u^{2} \partial_{u}^{2}\left(\phi^{(s-2)}+2 \partial_{u}^{2} \phi^{(s)}\right) .
$$

Finally, summing up the two contributions $(2.48,2.52)$, the $\epsilon^{(s-1)}$-variation of the bulk action plus the zeroth boundary term reads

$$
\delta_{\epsilon^{(s-1)}}\left(\mathcal{I}_{\mathcal{M}}+\mathcal{I}_{\partial \mathcal{M}}^{0^{\prime}}\right)=\frac{z_{B}^{-d}}{2}\left\langle\left\langle\epsilon^{(s-1)} \mid \mathrm{P}_{\mathrm{e}} z u \cdot \partial \zeta^{(s, s-2)}\right\rangle_{z_{B}} .\right.
$$

We now look for a local $\partial_{z} \phi^{(r)}$-independent expression whose variation is precisely (2.55).

Using an identity of P-operator (C.2) and an integration by parts, the variation (2.55) becomes

$$
-\frac{z_{B}^{-d}}{2}\left\langle\left\langle z\left(\partial_{u} \cdot \partial+u \cdot \partial \partial_{u}^{2}\right) \epsilon^{(s-1)} \mid \mathrm{P}_{\mathrm{o}} \zeta^{(s-2)}\right\rangle z_{B} .\right.
$$

So it can be cancelled by a term of the form

$$
\mathcal{I}_{\partial \mathcal{M}}^{1}=\frac{z_{B}^{-d}}{2}\left\langle\xi^{(s-2)} \mid \mathrm{P}_{\mathrm{o}} \zeta^{(s-2)}\right\rangle_{z_{B}}
$$

where $\xi^{(s-2)}$ is a linear combination of the fields which does not involve any $\partial_{z} \phi^{(r)}$ and whose $\epsilon^{(s-1)}$-variation is

$$
\delta_{\epsilon^{(s-1)}} \xi^{(s-2)}=z\left(\partial_{u} \cdot \partial+u \cdot \partial \partial_{u}^{2}\right) \epsilon^{(s-1)} .
$$

From the gauge transformations (2.18) of the decomposed fields, we determine $\xi^{(s-2)}$ as

$$
\xi^{(s-2)}=\frac{1}{2}\left(\partial_{u}^{2} \phi^{(s)}-\phi^{(s-2)}\right) .
$$

Now we turn to the determination of the second $\left(\epsilon^{(s-1)}\right.$-invariant) boundary term $\mathcal{I}_{\partial \mathcal{M}}^{2}$ by requiring (2.28) under the $\partial_{z} \epsilon^{(s-2)}$-variation. For that, let us first consider the vanishing boundary condition for the gauge parameter: $\epsilon^{(s-2)}\left(z_{B}\right)=0$, but with an arbitrary $\partial_{z} \epsilon^{(s-2)}\left(z_{B}\right)$. After finding $\mathcal{I}_{\partial \mathcal{M}}^{2}$ with this condition, we will see, in the next subsection, whether this boundary condition can be relaxed by adding another boundary term.

Since $\delta_{\epsilon^{(s-2)}} \mathcal{I}_{\mathcal{M}}=0$ with $\epsilon^{(s-2)}\left(z_{B}\right)=0$, we require simply

$$
\delta_{\epsilon^{(s-2)}} \mathcal{I}_{\partial \mathcal{M}}^{2}=-\delta_{\epsilon^{(s-2)}}\left(\mathcal{I}_{\partial \mathcal{M}}^{0^{\prime}}+\mathcal{I}_{\partial \mathcal{M}}^{1}\right)
$$


where the zeroth and the first boundary terms are obtained in the previous section as

$$
\mathcal{I}_{\partial \mathcal{M}}^{0^{\prime}}+\mathcal{I}_{\partial \mathcal{M}}^{1}=\frac{z_{B}^{-d}}{2}\left[\left\langle\left\langle\phi^{(s)} \mid \mathrm{P}_{\mathrm{e}}^{\prime} \chi^{(s)}\right\rangle_{z_{B}}+\left\langle\left\langle\psi^{(s-3)} \mid \mathrm{P}_{\mathrm{o}}^{\prime} \chi^{(s-3)}\right\rangle_{z_{B}}+\left\langle\left\langle\xi^{(s-2)} \mid \mathrm{P}_{\mathrm{o}} \zeta^{(s-2)}\right\rangle_{z_{B}}\right]\right.\right.\right.
$$

The above is written in terms of the boundary values of $\phi^{(s)}, \chi^{(s)}, \psi^{(s-3)}, \chi^{(s-3)}, \xi^{(s-2)}$ and $\zeta^{(s, s-2)}$, and their boundary values transform under $\epsilon^{(s-2)}$ with $\epsilon^{(s-2)}\left(z_{B}\right)=0$ as

$$
\begin{aligned}
\delta \phi^{(s)} & =0, & \delta \chi^{(s)} & =-3 u^{2} z \partial_{z} \epsilon^{(s-2)} \\
\delta \psi^{(s-3)} & =0, & \delta \chi^{(s-3)} & =z\left(3 \partial \cdot \partial_{u}+u \cdot \partial \partial_{u}^{2}\right) \partial_{z} \epsilon^{(s-2)}, \\
\delta \xi^{(s-2)} & =-z \partial_{z} \epsilon^{(s-2)}, & \delta \zeta^{(s-2)} & =2\left[3(d+2 s-5)+u^{2} \partial_{u}^{2}\right] z \partial_{z} \epsilon^{(s-2)} .
\end{aligned}
$$

By using the above transformations rules, we get the $\epsilon^{(s-2)}$-variation of the boundary term, with $\epsilon^{(s-2)}\left(z_{B}\right)=0$, as

$$
\delta_{\epsilon^{(s-2)}}\left(\mathcal{I}_{\partial \mathcal{M}}^{0^{\prime}}+\mathcal{I}_{\partial \mathcal{M}}^{1}\right)=-z_{B}^{-d}\left\langle\mathrm{P}_{\mathrm{o}} z \partial_{z} \epsilon^{(s-2)} \mid \zeta^{(s-2)}\right\rangle_{z_{B}}
$$

This variation can be compensated by the following second boundary term:

$$
\mathcal{I}_{\partial \mathcal{M}}^{2}=\frac{z_{B}^{-d}}{4}\left\langle\left\langle\zeta^{(s-2)} \mid \mathrm{P}_{\mathrm{o}}^{\prime \prime} \zeta^{(s-2)}\right\rangle_{z_{B}}, \quad \mathrm{P}_{\mathrm{o}}^{\prime \prime}:=\mathrm{P}_{\mathrm{o}}\left[3(d+2 s-5)+u^{2} \partial_{u}^{2}\right]^{-1} .\right.
$$

The inversion of the operator can be readily done and the result is

$$
\mathrm{T}:=\left[3(d+2 s-5)+u^{2} \partial_{u}^{2}\right]^{-1}=\frac{1}{3(d+2 s-5)} \sum_{n=0}^{\infty} \frac{1}{4^{n}\left(a_{+}\right)_{n}\left(a_{-}\right)_{n}}\left(u^{2}\right)^{n}\left(\partial_{u}^{2}\right)^{n},
$$

with

$$
a_{ \pm}=\frac{d+2 u \cdot \partial_{u}+2}{4} \pm \sqrt{\left(\frac{d+2 u \cdot \partial_{u}-2}{4}\right)^{2}+\frac{3(d+2 s-5)}{4}} .
$$

Notice that this second boundary term has a peculiar aspect. It contains, for $s>2$, a second order derivative term in $x$ (since $\zeta^{(s-2)}$ contains a term with one derivative).

It remains to examine whether the symmetries under $\epsilon^{(s-2)}\left(z_{B}\right)$ can be restored. For that we need first to obtain the variation of $\mathcal{I}_{\mathcal{M}}+\mathcal{I}_{\partial \mathcal{M}}$ under $\epsilon^{(s-2)}$, and it is given by (see appendix D for more details)

$$
\delta_{\epsilon^{(s-2)}}\left(\mathcal{I}_{\mathcal{M}}+\mathcal{I}_{\partial \mathcal{M}}\right)=\frac{z_{B}^{-d}}{2}\left\langle\left\langle\epsilon^{(s-2)} \mid J_{\mathcal{M}}+J_{\partial \mathcal{M}}\right\rangle_{z_{B}}\right.
$$


with

$$
\begin{aligned}
& J_{\mathcal{M}}+J_{\partial \mathcal{M}}=\mathrm{P}_{\mathrm{o}}\left\{z ^ { 2 } \left[2\left(\left(\partial_{u} \cdot \partial\right)^{2}-\square \partial_{u}^{2}\right)-\frac{5}{2} u \cdot \partial \partial_{u} \cdot \partial \partial_{u}^{2}-\frac{3}{2}(u \cdot \partial)^{2}\left(\partial_{u}^{2}\right)^{2}\right.\right. \\
&\left.+\frac{1}{2} u \cdot \partial\left(u \cdot \partial \partial_{u}^{2}+3 \partial_{u} \cdot \partial\right)\left(1+4 \mathrm{~T} u^{2} \partial_{u}^{2}\right) \partial_{u}^{2}\right] \phi^{(s)} \\
&+\left[\left(s-2+\frac{1}{2} u^{2} \partial_{u}^{2}\right)\left(3(d+2 s-5)+u^{2} \partial_{u}^{2}\right)\right. \\
&\left.\quad-\frac{1}{2}\left(7 d+10 s-19-u^{2} \partial_{u}^{2}\right) u^{2} \partial_{u}^{2}-4 \partial_{u}^{2}\left(u^{2}\right)^{2} \partial_{u}^{2} u^{2} \partial_{u}^{2} \mathrm{\top}\right] \partial_{u}^{2} \phi^{(s)} \\
&+z^{3} u \cdot \partial\left(u \cdot \partial+3 \partial_{u} \cdot \partial\right) \mathrm{T} u \cdot \partial \psi^{(s-3)} \\
&+\left.z\left[\left(d+2 s-5+u^{2} \partial_{u}^{2}\right) u \cdot \partial+u^{2} \partial_{u} \cdot \partial-2 \partial_{u}^{2}\left(u^{2}\right)^{2} \partial_{u}^{2} \mathrm{\top} u \cdot \partial\right] \psi^{(s-3)}\right\} \\
&-(d+2 s-5)\left[\left(3(s-2)+u^{2} \partial_{u}^{2}\right) \mathrm{P}_{\mathrm{e}} \partial_{u}^{2} \phi^{(s)}+z u \cdot \partial \mathrm{P}_{\mathrm{e}} \psi^{(s-3)}\right] .
\end{aligned}
$$

Notice that the variation does not contain $\phi^{(s-2)}$ while the dependence on $\phi^{(s-1)}$ is only through $\psi^{(s-3)}$. In the next section, we show that the variation (2.68) cannot be cancelled by including additional boundary terms.

Before closing this section let us assemble all the terms and give the complete boundary term

$$
\begin{aligned}
\mathcal{I}_{\partial \mathcal{M}}=\frac{z_{B}^{-d}}{2} & {\left[\left\langle\phi^{(s)} \mid \mathrm{P}_{\mathrm{e}}^{\prime} \chi^{(s)}\right\rangle_{z_{B}}+\frac{1}{2}\left\langle 3 \partial_{u}^{2} \phi^{(s-1)}+\phi^{(s-3)} \mid \mathrm{P}_{\mathrm{o}}^{\prime} \chi^{(s-3)}\right\rangle_{z_{B}}\right.} \\
& +\frac{1}{2}\left\langle\left\langle\partial_{u}^{2} \phi^{(s)}-\phi^{(s-2)} \mid \mathrm{P}_{\mathrm{o}} \zeta^{(s-2)}\right\rangle_{z_{B}}+\frac{1}{2}\left\langle\left\langle\zeta^{(s-2)} \mid \mathrm{P}_{\mathrm{o}}^{\prime \prime} \zeta^{(s, s-2)}\right\rangle_{z_{B}}\right],\right.
\end{aligned}
$$

where we recall the definitions

$$
\begin{aligned}
\chi^{(s)}:= & \left(z \partial_{z}+s-2\right) \phi^{(s)}-z(u \cdot \partial) \varphi^{(s, s-1)}-u^{2} \phi^{(s-2)}, \\
\chi^{(s-3)}:= & \frac{1}{2}\left(z \partial_{z}+s-3\right)\left(3 \partial_{u}^{2} \phi^{(s-1)}+\phi^{(s-3)}\right)+ \\
& \quad+(d+2 s-5) \phi^{(s-3)}+\frac{1}{2} u^{2} \partial_{u}^{2}\left(\phi^{(s-3)}+\partial_{u}^{2} \phi^{(s-1)}\right), \\
\zeta^{(s-2)}:= & \frac{1}{2} z u \cdot \partial\left(3 \partial_{u}^{2} \phi^{(s-1)}+\phi^{(s-3)}\right)+ \\
& +3(d+2 s-5) \phi^{(s-2)}+u^{2} \partial_{u}^{2}\left(\phi^{(s-2)}+2 \partial_{u}^{2} \phi^{(s)}\right) .
\end{aligned}
$$

\section{General boundary actions and anomalies}

In order to see whether it is possible to maintain the symmetry under $\epsilon^{(s-2)}\left(z_{B}\right)$ in the presence of a boundary we will proceed in two steps. First, we determine the most general action compatible with the equations of motion and the invariance under $\epsilon^{(s-1)}\left(z_{B}\right), \partial_{z} \epsilon^{(s-1)}\left(z_{B}\right)$ and $\partial_{z} \epsilon^{(s-2)}\left(z_{B}\right)$ and then we see whether this action can be invariant $\epsilon^{(s-2)}\left(z_{B}\right)$.

The most general action differs from $(2.69)$ by a quadratic term, $\mathcal{I}_{\partial \mathcal{M}}^{\text {(c.t.) }}$, with no $\partial_{z} \phi^{(r)}$ dependence and invariant under the transformations generated by $\epsilon^{(s-1)}\left(z_{B}\right), \partial_{z} \epsilon^{(s-1)}\left(z_{B}\right)$ and $\partial_{z} \epsilon^{(s-2)}\left(z_{B}\right)$. 
- Invariance under $\partial_{z} \epsilon^{(s-2)}\left(z_{B}\right)$ implies that $\mathcal{I}_{\partial \mathcal{M}}^{(\text {c.t. }}$ does not depend on $\phi^{(s-2)}$;

- invariance under $\partial_{z} \epsilon^{(s-1)}\left(z_{B}\right)$ is insured if the dependence on $\phi^{(s-1)}$ and $\phi^{(s-3)}$ is only through the combination $\psi^{(s-3)}$ or equivalently

$$
\beta^{(s-3)}:=\mathrm{\top} \psi^{(s-3)} .
$$

The latter has the advantage of a simple transformation under $\epsilon^{(s-1)}\left(z_{B}\right)$ :

$$
\delta_{\epsilon^{(s-1)}} \beta^{(s-3)}=\partial_{u}^{2} \epsilon^{(s-1)} .
$$

In the following, we examine all $\epsilon^{(s-1)}\left(z_{B}\right)$-invariants built in terms of $\phi^{(s)}$ and $\beta^{(s-3)}$. Since the gauge variation (2.68) contains up to two derivatives acting on $\phi^{(s)}$, we focus on the ones involving at most two derivatives on $\phi^{(s)}$.

\subsection{Gauge-invariant boundary terms and their transformations}

To examine all invariants, we classify the latter as the ones quadratic or linear in the basic invariants, which are linear in $\phi^{(s)}$ and $\beta^{(s-3)} .^{5}$ There are only two basic invariants up to two derivatives on $\phi^{(s)}$, and they are

$$
\begin{aligned}
A^{(s)} & :=z^{2} \mathcal{F}_{M^{d}} \phi^{(s)}-\frac{1}{2} z^{3}(u \cdot \partial)^{3} \beta^{(s-3)}, \\
C^{(s-4)} & :=\left(\partial_{u}^{2}\right)^{2} \phi^{(s)}-z\left(4 \partial_{u} \cdot \partial+u \cdot \partial \partial_{u}^{2}\right) \beta^{(s-3)},
\end{aligned}
$$

where $\mathcal{F}_{M^{d}}$ is the $d$-dimensional Fronsdal operator:

$$
\mathcal{F}_{M^{d}}:=\square-u \cdot \partial \partial_{u} \cdot \partial+\frac{1}{2}(u \cdot \partial)^{2} \partial_{u}^{2} \cdot
$$

Notice that these two invariants are related as

$$
\begin{aligned}
& \left(\partial_{u}^{2}\right)^{2} A^{(s)}=3 z^{2}\left[\square+u \cdot \partial \partial_{u} \cdot \partial+\frac{1}{6}(u \cdot \partial)^{2} \partial_{u}^{2}\right] C^{(s-4)}, \\
& z\left(\partial_{u} \cdot \partial-\frac{1}{2} u \cdot \partial \partial_{u}^{2}\right) A^{(s)}+\frac{1}{4} z^{3}(u \cdot \partial)^{3} C^{(s-4)}=0 .
\end{aligned}
$$

Hence, the double trace and the de Donder divergence of $A^{(s)}$ are given by $C^{(s-4)}$.

Concerning the quadratic terms built with these two invariants, there is only one invariant involving up to two derivatives:

$$
\mathcal{I}_{\partial \mathcal{M}}^{1(\text { c.t. })}=z_{B}^{-d}\left\langle\left\langle C^{(s-4)} \mid \mathcal{O} C^{(s-4)}\right\rangle_{z_{B}},\right.
$$

where $\mathcal{O}$ is a self-adjoint operator in $u$ and $x$ at most second-order in $z \partial$. The quadratic terms in $A^{(s)}$ contain at least four derivatives and the bilinear (cross) terms in $A^{(s)}$ and $C^{(s-4)}$ are either containing higher derivatives or re-expressible as (3.7) using (3.5). The explicit form $\mathcal{O}$ is to be determined by requiring the invariance of the total action under $\epsilon^{(s-2)}$.

\footnotetext{
${ }^{5}$ This is similar to the problem of unconstrained gauge invariance with a compensator examined in [52-54] and in particular the case $k=1$ in [53].
} 
The linear invariant in $A^{(s)}$ and $C^{(s-4)}$ is given by

$$
\mathcal{I}_{\partial \mathcal{M}}^{2 \text { (c.t.) }}=z_{B}^{-d}\left[\left\langle\left\langle\phi^{(s)} \mid G^{(s)}\right\rangle_{z_{B}}+\left\langle\left\langle\beta^{(s-3)} \mid K^{(s-3)}\right\rangle_{z_{B}}\right]\right.\right.
$$

where $G^{(s)}$ and $K^{(s-3)}$ are linear invariants so that, with the restriction of number of derivatives, they are given by $A^{(s)}$ and $C^{(s-4)}$. The gauge invariance of $\mathcal{I}_{\partial \mathcal{M}}^{2(\text { c.t. })}$ under $\epsilon^{(s-1)}$ imposes the Bianchi identities on these objects: ${ }^{6}$

$$
z \partial_{u} \cdot \partial G^{(s)}=u^{2} K^{(s-3)} .
$$

Using the identity (3.6), we find the solution for (3.9) as

$$
\begin{aligned}
& G^{(s)}=c\left[\left(1-\frac{1}{4} u^{2} \partial_{u}^{2}\right) A^{(s)}+\frac{1}{8} z^{2}(u \cdot \partial)^{2} u^{2} C^{(s-4)}\right], \\
& K^{(s-3)}=-c \frac{z}{4} \partial \cdot \partial_{u}\left[\partial_{u}^{2} A^{(s)}-\frac{1}{2} z^{2}(u \cdot \partial)^{2} C^{(s-4)}\right],
\end{aligned}
$$

where $c$ is an arbitrary constant.

We now calculate the variation of the total counterterm, $\mathcal{I}_{\partial \mathcal{M}}^{\text {(c.t.) }}=\mathcal{I}_{\partial \mathcal{M}}^{1 \text { (c.t.) }}+\mathcal{I}_{\partial \mathcal{M}}^{2 \text { (c.t.) }}$, under the remaining gauge transformation, that is, the one by $\epsilon^{(s-2)}\left(z_{B}\right)$. We obtain the variation of the counterterms as

$$
\delta_{\epsilon^{(s-2)}} \mathcal{I}_{\partial \mathcal{M}}^{\text {(c.t.) }}=\frac{z_{B}^{-d}}{2}\left\langle\left\langle\epsilon^{(s-2)} \mid J_{\partial \mathcal{M}}^{\text {(c.t.) }}\right\rangle_{z_{B}},\right.
$$

with

$$
\begin{aligned}
J_{\partial \mathcal{M}}^{(\text {c.t. })=-4[} & 4(d+2 s-6) u^{2}+\left(u^{2}\right)^{2} \partial_{u}^{2}+ \\
& \left.+z^{2}\left(3 u \cdot \partial+u^{2} \partial_{u} \cdot \partial\right) \mathrm{T}\left(4 u \cdot \partial+u^{2} \partial_{u} \cdot \partial\right)\right] \mathcal{O} C^{(s-4)} \\
+c\{ & 2(d+2 s-6) \partial_{u}^{2} A^{(s)}-z^{2}\left[(d+2 s-4)(u \cdot \partial)^{2}+u^{2}\left(2 \square+u \cdot \partial \partial_{u} \cdot \partial\right)\right] C^{(s-4)} \\
& \left.-z^{2}\left(u^{2} \partial_{u} \cdot \partial+3 u \cdot \partial\right) \mathrm{T} \partial_{u} \cdot \partial\left[\partial_{u}^{2} A^{(s)}-\frac{z^{2}}{2}(u \cdot \partial)^{2} C^{(s-4)}\right]\right\}
\end{aligned}
$$

For more details on the calculation, see appendix E. An important property of the variations of the invariants that we obtained is that the variation involves terms with different number of derivatives. The origin of this property lies in the transformation of $\phi^{(s)}$ and $\beta^{(s-3)}$ under $\epsilon^{(s-2)}$ with respectively zero and one derivative.

\subsection{Anomaly}

Now we determine the variation under $\epsilon^{(s-2)}$ of the total action:

$$
\delta_{\epsilon^{(s-2)}}\left(\mathcal{I}_{\mathcal{M}}+\mathcal{I}_{\partial \mathcal{M}}+\mathcal{I}_{\partial \mathcal{M}}^{(\text {c.t. })}\right)=\left\langle\left\langle\epsilon^{(s-2)} \mid \mathscr{A}^{(s-2)}\right\rangle_{z_{B}},\right.
$$

\footnotetext{
${ }^{6}$ Recall that the gauge transformation of $\phi^{(s)}$ is $z u \cdot \partial \epsilon^{(s-1)}$, this explains the $z$ factor in (3.9).
} 
and see whether there exists a choice for the counterterms such that this variation vanishes. In other words, we examine whether there exist an operator $\mathcal{O}(3.7)$ and constants $c$ such that the anomaly:

$$
\mathscr{A}^{(s-2)}:=\frac{z^{-d}}{2}\left(J_{\mathcal{M}}+J_{\partial \mathcal{M}}+J_{\partial \mathcal{M}}^{(\text {c.t. })}\right),
$$

vanishes. In the last section, we have shown that $J_{\partial \mathcal{M}}^{(\text {c.t.) }}$ is given in terms of $A^{(s)}$ and $C^{(s-4)}$. Actually, the gauge variation $J_{\mathcal{M}}+J_{\partial \mathcal{M}}(2.68)$ can also be expressed in terms of these invariants as

$$
\begin{aligned}
J_{\mathcal{M}}+J_{\partial \mathcal{M}}= & -\mathrm{P}_{\mathrm{o}} \partial_{u}^{2} A^{(s)}-\mathrm{P}_{\mathrm{o}} \frac{z^{2}}{2}\left[(u \cdot \partial)^{2}-4 u \cdot \partial\left(u \cdot \partial \partial_{u}^{2}+3 \partial_{u} \cdot \partial\right) \mathrm{T} u^{2}\right] C^{(s-4)} \\
- & \left\{\mathrm{P}_{\mathrm{o}}\left[s+2 d-\frac{1}{2}(3 d+8 s-18) u^{2} \partial_{u}^{2}+4 \partial_{u}^{2}\left(u^{2}\right)^{2} \partial_{u}^{2} \mathrm{~T}\right] u^{2}\right. \\
& \left.+(d+2 s-5)\left(\mathrm{P}_{\mathrm{e}} u^{2}+3(s-2) u^{2} \mathrm{P}_{\mathrm{o}}^{\prime}\right)\right\} C^{(s-4)}
\end{aligned}
$$

In order to see whether the anomaly can be canceled, let us first focus on the part of $\mathscr{A}^{(s-2)}$ proportional to the trace of $A^{(s)}$, then we get

$$
z^{-d}\left[-\frac{1}{2}+c\left(d+2 s-6-z^{2} \frac{\left(u^{2} \partial_{u} \cdot \partial+3 u \cdot \partial\right) \partial_{u} \cdot \partial}{6(d+2 s-5)}\right)\right] \partial_{u}^{2} A^{(s)} .
$$

For $s>2$ where $\partial_{u} \cdot \partial \partial_{u}^{2} A^{(s)}$ is not zero this term cannot be canceled by appropriately choosing $c$, and it is independent of $C^{(s-4)}$. Therefore, there is no boundary counterterm which leads to the cancellation of this term implying that the symmetry under $\epsilon^{(s-2)}$ cannot be restored.

Although the counterterm cannot restore the gauge symmetries, it can modify the anomaly $\mathscr{A}^{(s-2)}$. By focusing now on the part of $\mathscr{A}^{(s-2)}$ proportional to $C^{(s-4)}$, we get

$$
\begin{aligned}
& -z^{-d+2}\left\{\mathrm{P}_{\mathrm{o}}\left[\frac{1}{4}(u \cdot \partial)^{2}-u \cdot \partial\left(u \cdot \partial \partial_{u}^{2}+3 \partial_{u} \cdot \partial\right) \mathrm{T} u^{2}\right]\right. \\
& \left.+2\left(3 u \cdot \partial+u^{2} \partial_{u} \cdot \partial\right) \mathrm{T}\left(4 u \cdot \partial+u^{2} \partial_{u} \cdot \partial\right) \mathcal{O}\right\} C^{(s-4)} \\
& -\frac{z^{-d}}{2}\left\{\mathrm{P}_{\mathrm{o}}\left[s+2 d-\frac{1}{2}(3 d+8 s-18) u^{2} \partial_{u}^{2}+4 \partial_{u}^{2}\left(u^{2}\right)^{2} \partial_{u}^{2} \mathrm{~T}\right] u^{2}\right. \\
& \left.+(d+2 s-5)\left(\mathrm{P}_{\mathrm{e}} u^{2}+3(s-2) u^{2} \mathrm{P}_{\mathrm{o}}^{\prime}\right)+4\left[4(d+2 s-6) u^{2}+\left(u^{2}\right)^{2} \partial_{u}^{2}\right] \mathcal{O}\right\} C^{(s-4)},
\end{aligned}
$$

where we have chosen $c=0$. Notice that in the last two lines, we can factor out a $u^{2}$ so that they can be recast as

$$
\begin{aligned}
& -\frac{z^{-d}}{2} u^{2}\left[4\left[4(d+2 s-6)+u^{2} \partial_{u}^{2}\right] \mathcal{O}+(d+2 s-5)(d+2 s-7) \mathrm{P}_{\mathrm{o}}+\right. \\
& +(d+2 s-5) \mathrm{P}_{\mathrm{o}}^{\prime}\{2(d+2 s-3)-(d+2 s-8)(3 d+8 s-10) \\
& \left.\left.+4 u^{2} \partial_{u}^{2}-4(d+2 s-7) \partial_{u}^{2} \mathrm{\top} u^{2}\right\}\right] C^{(s-4)},
\end{aligned}
$$


where we have used the definition of $\mathrm{T}$ as well as the identities presented in appendix $\mathrm{C}$. It is important to note that (3.18) can be cancelled with a suitable choice of the operator $\mathcal{O}$. In the latter case, the anomaly, that is the gauge variation of the action under $\epsilon^{(s-2)}$, is given by

$$
\begin{aligned}
\mathscr{A}^{(s-2)}= & -\frac{z^{-d}}{2} \mathrm{P}_{\mathrm{o}} \partial_{u}^{2} A^{(s)}-z^{-d+2}\left\{\mathrm{P}_{\mathrm{o}}\left[\frac{1}{4}(u \cdot \partial)^{2}-u \cdot \partial\left(u \cdot \partial \partial_{u}^{2}+3 \partial_{u} \cdot \partial\right) \mathrm{T} u^{2}\right]\right. \\
& \left.+2\left(3 u \cdot \partial+u^{2} \partial_{u} \cdot \partial\right) \mathrm{T}\left(4 u \cdot \partial+u^{2} \partial_{u} \cdot \partial\right) \mathcal{O}\right\} C^{(s-4)},
\end{aligned}
$$

where the explicit form of $\mathcal{O}$ can be determined by requiring (3.18) to be zero. From the definitions of $A^{(s)}$ and $C^{(s-4)}$, one can see that the anomaly contains only terms proportional to $z^{-d+2}$ or $z^{-d+3}$ :

$$
\mathscr{A}^{(s-2)}=z^{-d+2} \mathcal{A}_{\phi} \phi^{(s)}+z^{-d+3} \mathcal{A}_{\psi} \psi^{(s-3)} .
$$

The absence of the terms proportional to $z^{-d}$ is due to the cancellation of (3.18), and this will have important consequences later in analyzing the anomaly of the finite par of the on-shell action.

Finally, the total action is given by

$$
\begin{aligned}
\mathcal{I}_{\mathcal{M}}+\mathcal{I}_{\partial \mathcal{M}} & +\mathcal{I}_{\partial \mathcal{M}}^{(c . t)}= \\
=-\int_{z_{B}}^{\infty} d z & \frac{z^{-d-1}}{2}\left\langle\varphi \mid\left(1-\frac{1}{4} U^{2} \partial_{U}^{2}\right) \mathcal{F} \varphi\right\rangle \\
+z_{B}^{-d}[ & \frac{1}{2}\left\langle\phi^{(s)} \mid \mathrm{P}_{\mathrm{e}}^{\prime} \chi^{(s)}\right\rangle_{z_{B}}+\frac{1}{4}\left\langle 3 \partial_{u}^{2} \phi^{(s-1)}+\phi^{(s-3)} \mid \mathrm{P}_{\mathrm{o}}^{\prime} \chi^{(s-3)}\right\rangle_{z_{B}} \\
& +\frac{1}{4}\left\langle\left\langle\partial_{u}^{2} \phi^{(s)}-\phi^{(s-2)} \mid \mathrm{P}_{\mathrm{o}} \zeta^{(s-2)}\right\rangle_{z_{B}}+\frac{1}{4}\left\langle\left\langle\zeta^{(s-2)} \mid \mathrm{P}_{\mathrm{o}}^{\prime \prime} \zeta^{(s, s-2)}\right\rangle_{z_{B}}\right.\right. \\
& +\left\langle C^{(s-4)} \mid \mathcal{O} C^{(s-4)}\right\rangle_{z_{B}}+\left\langle\left\langle\phi^{(s)} \mid G^{(s)}\right\rangle_{z_{B}}+\left\langle\left\langle\beta^{(s-3)} \mid K^{(s-3)}\right\rangle_{z_{B}}\right] .\right.
\end{aligned}
$$

\section{On-shell action}

The AdS/CFT corespondance, in the semi-classical regime, is the identification between the finite parts of the effective action $W_{\Lambda}[h]$ of the CFT and the on-shell HS action $S_{z_{B}}[h]$. The effective action, a functional of a spin $s$ field $h^{(s)}$, is invariant under the gauge symmetry $\delta h^{(s)}=u \cdot \partial \lambda^{(s-1)}$ and may be anomalous under the conformal transformation $\delta h^{(s)}=$ $-u^{2} \sigma^{(s-2)}$. In this section, we shall use our previous results in order to evaluate the HS action when the field is on-shell.

First, we have to make precise the AdS boundary conditions and how they are given in terms of $h^{(s)}$. This not straightforward, since as we saw, in the bulk of AdS we have the field $\phi^{(s-r)}$, with $r=0,1,2,3$ subject to the gauge transformations (2.18). For $s=2$, the standard procedure is to fix the gauge to the radial one, that is $\phi^{(1)}=\phi^{(0)}=0$ and to identify $h^{(2)}$ as the boundary value of $\phi^{(2)}$ at the origin. This prescription is not easily generalized to higher spins. In fact, the gauge parameters can set to zero only $\phi^{(s-1)}$ and $\phi^{(s-2)}$ but not the remaining one $\phi^{(s-3)}$. So we have to proceed differently. 
Using the gauge transfomations and the equations of motion, it is possible to reach a Coulomb-like (or synchronous-like) gauge, that is

$$
\partial_{u}^{2} \phi^{(s)}=\partial_{u} \cdot \partial \phi^{(s)}=0, \quad \phi^{(s-1)}=\phi^{(s-2)}=\phi^{(s-3)}=0 .
$$

In this gauge, the equations of motion simplify considerably and reduce to

$$
\left[\left(z \partial_{z}\right)^{2}-d z \partial_{z}-m_{s}^{2}-s+z^{2} \square\right] \phi^{(s)}=0
$$

which can be solved as

$$
\phi^{(s)}(z, x)=z^{2-s} U_{\frac{d+2 s-4}{2}}(z \sqrt{-\square}) h_{\mathrm{TT}}^{(s)}(x),
$$

where $h_{\mathrm{TT}}^{(s)}$ is a transverse and traceless $d$-dimensional spin $s$ field:

$$
\partial_{u}^{2} h_{\mathrm{TT}}^{(s)}=0, \quad \partial_{u} \cdot \partial h_{\mathrm{TT}}^{(s)}=0,
$$

and the Bessel-like function $U_{\nu}(z)$ is defined in (C.3) of appendix C. So we obtain the solution as a function of the boundary field $h_{\mathrm{TT}}^{(s)}$. The latter cannot be identified directly with $h^{(s)}$ because of the transverse and traceless properties of $h_{\mathrm{TT}}^{(s)}$. Since $h^{(s)}$ has a gauge invariance, we can identify it with $h_{\mathrm{TT}}^{(s)}$ up to a gauge and conformal transformation as

$$
h_{\mathrm{TT}}^{(s)}=h^{(s)}-u \cdot \partial \bar{\rho}^{(s-1)}\left[h^{(s)}\right]+u^{2} \rho^{(s-2)}\left[h^{(s)}\right],
$$

where the traceless $\bar{\rho}^{(s-1)}\left[h^{(s)}\right]$ and $\rho^{(s-2)}\left[h^{(s)}\right]$ are given in terms of the trace and the divergence of $h^{(s)}$ as is shown in the appendix $\mathrm{F}$.

The general solution can now be written by performing a general gauge transformation as

$$
\begin{aligned}
\phi^{(s)} & =z^{2-s} U_{\frac{d+2 s-4}{2}}(z \sqrt{-\square}) h_{\mathrm{TT}}^{(s)}+z(u \cdot \partial) \epsilon^{(s-1)}-u^{2} \epsilon^{(s-2)}, \\
\phi^{(s-1)} & =\left(z \partial_{z}+s-1+u^{2} \partial_{u}^{2}\right) \epsilon^{(s-1)}+z(u \cdot \partial) \epsilon^{(s-2)}, \\
\phi^{(s-2)} & =-z(u \cdot \partial) \partial_{u}^{2} \epsilon^{(s-1)}+\left[2\left(z \partial_{z}+s-2\right)+u^{2} \partial_{u}^{2}\right] \epsilon^{(s-2)}, \\
\phi^{(s-3)} & =-\left[3\left(z \partial_{z}+s-3\right)+u^{2} \partial_{u}^{2}\right] \partial_{u}^{2} \epsilon^{(s-1)}-z(u \cdot \partial) \partial_{u}^{2} \epsilon^{(s-2)} .
\end{aligned}
$$

Now, if we choose the gauge parameters as

$$
\begin{aligned}
& \epsilon^{(s-1)}=z^{1-s} U_{\frac{d+2 s-4}{2}}(z \sqrt{-\square}) \bar{\rho}^{(s-1)}\left[h^{(s)}\right], \\
& \epsilon^{(s-2)}=z^{2-s} U_{\frac{d+2 s-4}{2}}(z \sqrt{-\square}) \rho^{(s-2)}\left[h^{(s)}\right],
\end{aligned}
$$

then the solution can be expressed in terms of $h^{(s)}$ as

$$
\begin{aligned}
\phi^{(s)} & =z^{2-s} U_{\frac{d+2 s-4}{2}}(z \sqrt{-\square}) h^{(s)}, \\
\phi^{(s-1)} & =z^{2-s} \partial_{z} U_{\frac{d+2 s-4}{2}}(z \sqrt{-\square}) \bar{\rho}^{(s-1)}\left[h^{(s)}\right]+z^{3-s} U_{\frac{d+2 s-4}{2}}(z \sqrt{-\square}) u \cdot \partial \rho^{(s-2)}\left[h^{(s)}\right], \\
\phi^{(s-2)} & =z^{2-s}\left(2 z \partial_{z}+u^{2} \partial_{u}^{2}\right) U_{\frac{d+2 s-4}{2}}(z \sqrt{-\square}) \rho^{(s-2)}\left[h^{(s)}\right], \\
\phi^{(s-3)} & =-z^{3-s} U_{\frac{d+2 s-4}{2}}(z \sqrt{-\square}) u \cdot \partial \partial_{u}^{2} \rho^{(s-2)}\left[h^{(s)}\right],
\end{aligned}
$$

where we used the tracelessness of $\bar{\rho}^{(s-1)}\left[h^{(s)}\right]$. Now, our proposition for $S\left[h^{(s)}\right]$ is to evaluate $\mathcal{I}[\varphi]=\mathcal{I}\left[\phi^{(s)}, \phi^{(s-1)}, \phi^{(s-2)}, \phi^{(s-3)}\right]$ on the above solution which generates a functional of $h^{(s)}$. 


\subsection{Symmetries and anomalies of the on-shell action}

In this and the following subsections we examine the properties of the on-shell action:

$$
S_{z_{B}}\left[h^{(s)}\right]:=\left(\mathcal{I}_{\mathcal{M}}+\mathcal{I}_{\partial \mathcal{M}}+\mathcal{I}_{\partial \mathcal{M}}^{(\text {c.t. }}\right)\left[\phi^{(r)}\left[h^{(s)}\right]\right] .
$$

Let us first note that it has an expansion in $z_{B}$ :

$$
S_{z_{B}}=\sum_{n=3-s}^{[d / 2]} z_{B}^{2 n-d} S_{n}+S_{\text {fin }}+\log \left(\mu z_{B}\right) S_{\log }+o\left(z_{B}\right),
$$

where the logarithmic terms are given with a constant $\mu$ of mass dimension and they are absent for odd dimensions, $o\left(z_{B}\right)$ vanishes for small $z_{B}$ faster than $z_{B}$. Of particular importance are the finite (or renormalized) part $S_{\text {fin }}$ and logarithmic part $S_{\log }$. The CFT effective action $W_{\Lambda}$ has a similar expansion in terms of the cut-off $\Lambda$, the renormalized part $W_{\text {fin }}$ should, by the holographic correspondence, be equal (up to possible local counterterms) to $S_{\text {fin }}$. The AdS isometry $\left(z^{\prime}, x^{\prime}\right)=(\Omega z, \Omega x)$, under which $h^{(s)}$ transforms as

$$
h^{(s)}(x) \quad \rightarrow \quad h^{\prime(s)}(x)=\Omega^{2-s} h^{(s)}\left(\Omega^{-1} x\right),
$$

implies that $S_{\Omega z_{B}}\left[h^{\prime(s)}\right]=S_{z_{B}}\left[h^{(s)}\right]$. Combined with (4.10), this in turn gives the dilatation transformations of $S_{n}$ as

$$
S_{n}\left[h^{\prime(s)}\right]=\Omega^{2 n-d} S_{n}\left[h^{(s)}\right] .
$$

For odd $d$, the relation holds also for $S_{\text {fin }}$, whereas for even $d$ where logarithmic terms are also present we get

$$
S_{\log }\left[h^{\prime(s)}\right]=S_{\log }\left[h^{(s)}\right], \quad S_{\text {fin }}\left[h^{\prime(s)}\right]+\log \Omega S_{\log }\left[h^{\prime(s)}\right]=S_{\text {fin }}\left[h^{(s)}\right] .
$$

Next, let us examine the transformation under the gauge symmetries. Under a gauge transformation $\delta h^{(s)}=u \cdot \partial \bar{\lambda}^{(s-1)}-u^{2} \sigma^{(s-2)}$, with arbitrary $\sigma^{(s-2)}$ and traceless $\bar{\lambda}^{(s-1)}$, we have

$$
\delta \bar{\rho}^{(s-1)}\left[h^{(s)}\right]=\bar{\lambda}^{(s-1)}, \quad \delta \rho^{(s-2)}\left[h^{(s)}\right]=\sigma^{(s-2)} .
$$

Hence, from (4.7) one can find that the on-shell action is invariant under $\bar{\lambda}^{(s-1)}$ as it should, while under a conformal transformation by $\sigma^{(s-2)}$, we have

$$
\delta_{\sigma} S_{z_{B}}=\left\langle\left\langle\epsilon^{(s-2)} \mid \mathscr{A}^{(s-2)}\right\rangle_{z_{B}},\right.
$$

where $\epsilon^{(s-2)}=z^{2-s} U_{\frac{d+2 s-4}{2}}(z \sqrt{-\square}) \sigma^{(s-2)}$ and $\mathscr{A}^{(s-2)}(3.19)$ is now expressed in terms of $h^{(s)}$. Its explicit expression is rather cumbersome, let us just examine the $z_{B}$ dependence of $\delta_{\sigma} S_{z_{B}}$. In order to do that, we need the explicit dependence of $\mathscr{A}^{(s-2)}$ on $z_{B}$ which we write, from (3.20) and (4.8), as

$$
\mathscr{A}^{(s-2)}=z_{B}^{-d-s+4} U_{\frac{d+2 s-4}{2}}\left(z_{B} \sqrt{-\square}\right)\left(\mathscr{A}_{\phi}^{(s-2)}+z_{B}^{2} \mathscr{A}_{\psi}^{(s-2)}\right),
$$

where $\mathscr{A}_{\phi}^{(s-2)}$ and $\mathscr{A}_{\psi}^{(s-2)}$ are given from (3.20) by

$$
\mathscr{A}_{\phi}^{(s-2)}=\mathcal{A}_{\phi} h^{(s)}, \quad \mathscr{A}_{\psi}^{(s-2)}=\frac{1}{2} \mathcal{A}_{\psi}\left(3 \partial_{u} \cdot \partial+u \cdot \partial \partial_{u}^{2}\right) \rho^{(s-2)}\left[h^{(s)}\right] .
$$


Collecting all terms depending on $z_{B}$ in (4.15) we get

$$
\delta_{\sigma} S_{z_{B}}=z_{B}^{-d-2 s+6}\left\langle\left\langle\sigma^{(s-2)} \mid\left[U_{\frac{d+2 s-4}{2}}\left(z_{B} \sqrt{-\square}\right)\right]^{2}\left(\mathscr{A}_{\phi}^{(s-2)}+z_{B}^{2} \mathscr{A}_{\psi}^{(s-2)}\right)\right\rangle .\right.
$$

Using the expansion of $U_{\nu}(z)$ given in appendix $\mathrm{C}$, we see that for odd $d$ (that is for half integer $\nu$ ) there is no contribution proportional to $\left(z_{B}\right)^{0}$, similarly for even $d$ (or integer $\nu)$ there is no contribution proportional to $\log \left(\mu z_{B}\right)$. We conclude that

$$
\delta_{\sigma} S_{\mathrm{fin}}=0 \quad[d: \text { odd }], \quad \delta_{\sigma} S_{\log }=0 \quad[d: \text { even }] .
$$

Notice that this property is directly related to the counterm which removed the $z^{-d_{-}}$ independent part of the anomaly $\mathscr{A}^{(s-2)}(3.19)$.

The explicit expression for the anomaly (4.17) in terms of $h^{(s)}$ simplifies for low spins, for instance the $s=3$ anomaly is easily obtained after we first determine $\rho^{(1)}\left[h^{(3)}\right]$ as

$$
\rho^{(1)}\left[h^{(3)}\right]=-\frac{1}{4(d+1) \square}\left(1-\frac{d-2}{3 d} \frac{u \cdot \partial \partial_{u} \cdot \partial}{\square}\right) \partial_{u}^{2} \mathcal{F}_{M^{d}} h^{(3)},
$$

the anomaly is then given by

$$
\mathscr{A}_{\phi}^{(1)}=-\frac{1}{2} \partial_{u}^{2} \mathcal{F}_{M^{d}} h^{(3)}, \quad \mathscr{A}_{\psi}^{(1)}=-\frac{1}{4 d(d+1)} u \cdot \partial \partial_{u} \cdot \partial \partial_{u}^{2} \mathcal{F}_{M^{d}} h^{(3)} .
$$

\subsection{Spin 2 case}

In this subsection, we give the explicit expression for the on-shell action as a functional of the conformal field $h^{(2)}$. We then compare it with the on-shell action computed in the radial gauge. The two on-shell actions, as we will see, coincide.

As we showed in the previous section, the addition of a local couterterm allows the recovery of the full bulk gauge symmetry if $d>2$. When expresssed in terms of the transverse and traceless field $h_{\mathrm{TT}}^{(2)}$, the action reduces to

$$
S_{z_{B}}=-\frac{z_{B}^{-d}}{2}\left\langle\left\langle\phi^{(2)}\left[h_{\mathrm{TT}}^{(2)}\right] \mid z \partial_{z} \phi^{(2)}\left[h_{\mathrm{TT}}^{(2)}\right]\right\rangle_{z_{B}}+\frac{z_{B}^{-d+2}}{2(d-2)}\left\langle\phi^{(2)}\left[h_{\mathrm{TT}}^{(2)}\right] \mid \square \phi^{(2)}\left[h_{\mathrm{TT}}^{(2)}\right]\right\rangle z_{B},\right.
$$

where the traces and divergences of $\phi^{(2)}\left[h_{\mathrm{TT}}^{(2)}\right]$ vanish so do not contribute, and the second term comes from the counterterm (2.42). Using the explicit form of solutions (4.6) and the identity (C.9), one gets

$$
S_{z_{B}}=-\frac{z_{B}^{-d+4}}{2(d-2)}\left\langle h_{\mathrm{TT}}^{(2)} \mid V_{\frac{d-2}{2}}\left(z_{B} \sqrt{-\square}\right) \square^{2} h_{\mathrm{TT}}^{(2)}\right\rangle,
$$

with

$$
V_{\nu}(z):=\frac{1}{z^{2}} U_{\nu+1}(z)\left[U_{\nu}(z)-U_{\nu+1}(z)\right]=-\frac{1}{4 \nu(\nu-1)} U_{\nu+1}(z) U_{\nu-1}(z) .
$$

The expression of $h_{\mathrm{TT}}^{(2)}$ in terms of $h^{(2)}$ can be deduced as the general case presented in the appendix F, or alternatively by applying first the Fronsdal operator to the definition (4.5) to get

$$
\mathcal{F}_{M^{d}} h_{\mathrm{TT}}^{(2)}=\mathcal{F}_{M^{d}} h^{(2)}+\left[u^{2} \square+(d-2)(u \cdot \partial)^{2}\right] \rho^{(0)}\left[h^{(2)}\right],
$$


and then taking the trace of the equation to determine $\rho^{(0)}\left[h^{(2)}\right]$ as

$$
\rho^{(0)}\left[h^{(2)}\right]=-\frac{1}{4(d-1) \square} \partial_{u}^{2} \mathcal{F}_{M^{d}} h^{(2)} .
$$

Similarly, $\rho^{(1)}\left[h^{(2)}\right]$ can be obtained by first taking the trace of $(4.5)$ which determines $\partial_{u} \cdot \partial \rho^{(1)}\left[h^{(2)}\right]$ and then the divergence of (4.5) to get

$$
\rho^{(1)}\left[h^{(2)}\right]=\frac{1}{\square^{2}}\left[\square \partial_{u} \cdot \partial-\frac{1}{2(d-1)} \square u \cdot \partial \partial_{u}^{2}-\frac{d-2}{2(d-1)} u \cdot \partial\left(\partial_{u} \cdot \partial\right)^{2}\right] h^{(2)} .
$$

Finally, $h_{\mathrm{TT}}^{(2)}$ is expressed in terms of $h^{(2)}$ in the manifestly gauge invariant way as

$$
h_{\mathrm{TT}}^{(2)}=\mathcal{P}_{\mathrm{TT}}^{(2)} h^{(2)}, \quad \mathcal{P}_{\mathrm{TT}}^{(2)}=\left[1-\frac{1}{4(d-1)} u^{2} \partial_{u}^{2}-\frac{d-2}{4(d-1)} \frac{(u \cdot \partial)^{2}}{\square} \partial_{u}^{2}\right] \frac{\mathcal{F}_{M^{d}}}{\square},
$$

where $\mathcal{P}_{\mathrm{TT}}^{(2)}$ is the traceless and transverse projector of spin 2 field. Finally, the on-shell action as a functional of $h^{(2)}$ can be deduced as

$$
S_{z_{B}}=-\frac{z_{B}^{-d+4}}{2(d-2)}\left\langle\left\langle h^{(2)} \mid V_{\frac{d-2}{2}}\left(z_{B} \sqrt{-\square}\right) \mathcal{C}^{(2)} h^{(2)}\right\rangle\right.
$$

where $\mathcal{C}^{(2)}$ is given by a local differential operator:

$$
\mathcal{C}^{(2)}:=\square^{2} \mathcal{P}_{\mathrm{TT}}^{(2)} .
$$

Notice that, for $d \geq 4$, this result can be re-expressed in terms of the Weyl tensor $\mathscr{C}^{(2)}$ by making use of the identity:

$$
\left\langle\mathscr{C}^{(2)} \mid \mathscr{C}^{(2)}\right\rangle=6 \frac{d-3}{d-2}\left\langle h^{(2)} \mid \mathcal{C}^{(2)} h^{(2)}\right\rangle .
$$

We now compare with the radial gauge treatment which is valid for spin 2 . The solution can be readily determined as

$$
\phi^{(2)}=U_{\frac{d}{2}}(z \sqrt{-\mathcal{H}}) h^{(2)}, \quad \phi^{(1)}=\phi^{(0)}=0,
$$

where

$$
\mathcal{H}:=\left(1-\frac{1}{4(d-1)} u^{2} \partial_{u}^{2}\right) \mathcal{F}_{M^{d}}
$$

The operator $\mathcal{H}$ satisfies

$$
\left(\partial_{u} \cdot \partial-u \cdot \partial \partial_{u}^{2}\right) \mathcal{H}=0, \quad \partial_{u} \cdot \partial \mathcal{H}^{2}=0, \quad \partial_{u}^{2} \mathcal{H}^{2}=0,
$$

and, as a result:

$$
\mathcal{H}^{n+2}=\square^{n} \mathcal{H}^{2}, \quad[n \in \mathbb{N}] .
$$

The on-shell action with the radial gauge solution (4.32) can now be determined as

$$
\begin{aligned}
S_{z_{B}}= & -\frac{z_{B}^{-d}}{2}\left\langle\left\langle\phi^{(2)}\left[h^{(2)}\right] \mid\left(1-\frac{1}{2} u^{2} \partial_{u}^{2}\right) z \partial_{z} \phi^{(2)}\left[h^{(2)}\right]\right\rangle_{z_{B}}\right. \\
& +\frac{z_{B}^{-d+2}}{2(d-2)}\left\langle\left\langle\phi^{(2)}\left[h^{(2)}\right] \mid\left(1-\frac{1}{4} u^{2} \partial_{u}^{2}\right) \mathcal{F}_{M^{d}} \phi^{(2)}\left[h^{(2)}\right]\right\rangle_{z_{B}} .\right.
\end{aligned}
$$


By making use of the $z$-derivative of the solution:

$$
z \partial_{z} \phi^{(2)}=\frac{1}{d-2} z^{2} \mathcal{H} U_{\frac{d-2}{2}}(z \sqrt{-\mathcal{H}}) h^{(2)},
$$

and the identity (4.34) and

$$
\left\langle\mathcal{F}_{M^{d}} h^{(2)} \mid\left(1-\frac{d}{8(d-1)} u^{2} \partial_{u}^{2}\right) \mathcal{F}_{M^{d}} h^{(2)}\right\rangle=\left\langle\left\langle h^{(2)} \mid \mathcal{C}^{(2)} h^{(2)}\right\rangle,\right.
$$

one can show that the on-shell action agrees with (4.29).

Notice that this on-shell action (4.29) is invariant under gauge and Weyl symmetry, $\delta h^{(s)}=u \cdot \partial \lambda^{(s-1)}-u^{2} \sigma^{(s-2)}$, and notice also that all the divergent terms in the small $z_{B}$ limit are local. Of particular interest is the finite term for odd $d$ and the logarithmical divergent term for even $d$. Using the expansion of $U_{\nu}(z)$ given in appendix $\mathrm{C}$, the finite part of the on-shell action for odd $d$ is given by

$$
S_{\text {fin }}=\frac{\pi(-1)^{\frac{d+1}{2}}}{2^{4} \Gamma^{2}\left(\frac{d}{2}\right)}\left\langle h^{(2)} \mid\left(-\frac{1}{4} \square\right)^{\frac{d-4}{2}} \mathcal{C}^{(2)} h^{(2)}\right\rangle,
$$

and the logarithmically divergent part for even $d$ by

$$
S_{\log }=\frac{(-1)^{\frac{d+2}{2}}}{2^{3} \Gamma^{2}\left(\frac{d}{2}\right)}\left\langle\left\langle h^{(2)} \mid\left(-\frac{1}{4} \square\right)^{\frac{d-4}{2}} \mathcal{C}^{(2)} h^{(2)}\right\rangle .\right.
$$

\subsection{General case}

The on-shell action reduces, as before, to the boundary term $\mathcal{I}_{\partial \mathcal{M}}+\mathcal{I}_{\partial \mathcal{M}}^{\text {(c.t.) }}(2.69)$ where the fields should be replaced with the solution (4.8). The $z_{B}$ expansion of the action is obtained from the expansion of $U_{\nu}(z)$. The latter is of the form:

$$
U_{\nu}(z)=P_{\nu}\left(z^{2}\right)+z^{2 \nu}\left\{\begin{array}{cc}
(-1)^{-\frac{1}{2}} \frac{\pi}{2} Q_{\nu}\left(z^{2}\right) & {\left[\nu \in \mathbb{N}+\frac{1}{2}\right]} \\
\log z Q_{\nu}\left(z^{2}\right)+R_{\nu}\left(z^{2}\right) & {[\nu \in \mathbb{N}]}
\end{array}\right.
$$

where $P_{\nu}, Q_{\nu}$ and $R_{\nu}$ are power series.

The HS-Weyl-invariant part of the on-shell action, that is $S_{\text {fin }}$ for odd $d$ and $S_{\log }$ for even $d$, can be deduced easily from the one evaluated with the $h_{\mathrm{TT}}^{(s)}$ solution (4.6):

$$
S_{z_{B}}\left[h_{\mathrm{TT}}^{(s)}\right]=\frac{z_{B}^{-d-2 s+6}}{2(d+2 s-6)}\left\langle h_{\mathrm{TT}}^{(s)} \mid U_{\frac{d+2 s-4}{2}}\left(z_{B} \sqrt{-\square}\right) U_{\frac{d+2 s-6}{2}}\left(z_{B} \sqrt{-\square}\right) \square h_{\mathrm{TT}}^{(s)}\right\rangle .
$$

One can see from (4.40) that the finite part or logarithmically divergent part of (4.41) is given by the $Q_{\frac{d+2 s-4}{2}}(0) P_{\frac{d+2 s-6}{2}}(0)$ term. The finite part for odd $d$ is

$$
S_{\text {fin }}\left[h_{\mathrm{TT}}^{(s)}\right]=S_{\mathrm{fin}}\left[h^{(s)}\right]=\frac{\pi(-1)^{\frac{d+2 s-3}{2}}}{2^{2 s} \Gamma^{2}\left(\frac{d+2 s-4}{2}\right)}\left\langle h^{(s)} \mid\left(-\frac{1}{4} \square\right)^{\frac{d-4}{2}} \mathcal{C}^{(s)} h^{(s)}\right\rangle,
$$

and a similar expression for the logarithmically divergent term for even $d$ :

$$
S_{\log }\left[h_{\mathrm{TT}}^{(s)}\right]=S_{\log }\left[h^{(s)}\right]=\frac{(-1)^{\frac{d+2 s-2}{2}}}{2^{2 s-1} \Gamma^{2}\left(\frac{d+2 s-4}{2}\right)}\left\langle h^{(s)} \mid\left(-\frac{1}{4} \square\right)^{\frac{d-4}{2}} \mathcal{C}^{(s)} h^{(s)}\right\rangle,
$$


where $\mathcal{C}^{(s)}$ is a local differential operator given with the traceless and transverse projector $\mathcal{P}_{\mathrm{TT}}^{(s)}(\mathrm{F} .13)$ by

$$
\mathcal{C}^{(s)}:=(-\square)^{s} \mathcal{P}_{\mathrm{TT}}^{(s)} .
$$

To derive the above results, we have used the fact that $\delta_{\sigma} S_{\text {fin }}=0$ for odd $d$ and $\delta_{\sigma} S_{\log }=0$ for even $d$. As in the spin 2 case, these results can be re-expressed for $d \geq 4$ in terms of the spin $s$ Weyl tensor $\mathscr{C}^{(s)}$ since

$$
\left\langle\mathscr{C}^{(s)} \mid \mathscr{C}^{(s)}\right\rangle \propto\left\langle\left\langle h^{(s)} \mid \mathcal{C}^{(s)} h^{(s)}\right\rangle .\right.
$$

See appendix F for more details.

For low spins the explicit expression is easily obtained. We give here the complete expression for spin 3 in a manifestly gauge invariant way. The projector $\mathcal{P}_{\mathrm{TT}}^{(3)}$ is given by

$$
\begin{aligned}
\mathcal{P}_{\mathrm{TT}}^{(3)}=[1 & -\frac{d}{4(d+1)} \frac{(u \cdot \partial)^{2}}{\square} \partial_{u}^{2}+\frac{d-2}{12(d+1)} \frac{(u \cdot \partial)^{3} \partial_{u} \cdot \partial}{\square^{2}} \partial_{u}^{2}-\frac{1}{4(d+1)} u^{2} \partial_{u}^{2} \\
& \left.+\frac{1}{4(d+1)} u^{2} \frac{u \cdot \partial \partial_{u} \cdot \partial}{\square} \partial_{u}^{2}\right] \frac{\mathcal{F}_{M^{d}}}{\square}
\end{aligned}
$$

and the on-shell action can be calculated using the solution (4.6) as

$$
\begin{aligned}
S_{z_{B}}= & \frac{z_{B}^{-d}}{2 d}\left\langle\left\langle h^{(3)} \mid U_{\frac{d+2}{2}}\left(z_{B} \sqrt{-\square}\right) U_{\frac{d}{2}}\left(z_{B} \sqrt{-\square}\right) \frac{1}{\square^{2}} \mathcal{C}^{(3)} h^{(3)}\right\rangle\right. \\
& +\frac{z_{B}^{-d+2}}{8(d+1)}\left\langle\left\langle\partial_{u}^{2} \mathcal{F}_{M^{d}} h^{(3)}\right|\left[U_{\frac{d+2}{2}}\left(z_{B} \sqrt{-\square}\right)\right]^{2}\left(1-\frac{d-2}{3 d} \frac{u \cdot \partial \partial_{u} \cdot \partial}{\square}\right)\right. \\
& \left.\left(1+\frac{z_{B}^{2} u \cdot \partial \partial_{u} \cdot \partial}{2 d(d+1)}\right) \partial_{u}^{2} \mathcal{F}_{M^{d}} h^{(3)}\right\rangle .
\end{aligned}
$$

The first line is invariant under the conformal transformation and the second line reproduces the anomaly.

Notice that the on-shell action $S_{z_{B}}$ (4.47) is given by a linear combination of tensor structures whose coefficients are functions of the form $U_{\nu}(z) U_{\nu^{\prime}}(z)$ with $z=z_{B} \sqrt{-\square}$. In the expansion of the latter, only the terms compensating the overall power of $z_{B}$ contribute to the finite or logarithmically divergent part. This pattern is general for arbitrary spins, and one can see from (4.40) that $S_{\text {fin }}$ (odd $d$ ) or $S_{\log }$ (even $d$ ) is determined by the $(-1)^{-\frac{1}{2}} \frac{\pi}{2} z^{2 \nu} Q_{\nu} P_{\nu^{\prime}}$ or $z^{2 \nu} \log z Q_{\nu} P_{\nu^{\prime}}$ term respectively. Therefore, they share the same tensor structure differing only by a factor of $(-1)^{-\frac{1}{2}} \frac{\pi}{2}$, and they are both invariant as we have shown in this section. In the case of $S_{\text {fin }}$ for even $d$, the situation is rather different and the relevant term in the expansion of $U_{\nu} U_{\nu^{\prime}}$ is not only $z^{2 \nu} \log z Q_{\nu} P_{\nu^{\prime}}$ but also $P_{\nu} P_{\nu^{\prime}}$ and $z^{2 \nu} R_{\nu} P_{\nu^{\prime}}{ }^{7}$ The latter two terms being associated with different tensor structures are responsible for the anomaly. However, they give local contributions to the on-shell action since they do not involve any term with $\log \sqrt{-\square}$ or odd powers of $\sqrt{-\square}$. Consequently, the non-local part of $S_{\text {fin }}$ (even $d$ ) has a similar form as (4.43) with an insertion of $\log \sqrt{-\square}$.

\footnotetext{
${ }^{7}$ The remaining terms $z^{2\left(\nu+\nu^{\prime}\right)} \log z Q_{\nu} R_{\nu^{\prime}}$ and $z^{2\left(\nu+\nu^{\prime}\right)}(\log z)^{2} Q_{\nu} Q_{\nu^{\prime}}$ contribute to the on-shell action with terms which vanish in the limit $z_{B} \rightarrow 0$.
} 


\section{Conclusion}

In this paper we have determined the on-shell action for a free higher-spin gauge field in AdS. In order to accomplish this we first determined the boundary terms which are necessary to reproduce the equation of motion in the presence of a finite distance boundary. We constrained the boundary terms with the requirement of invariance under the symmetry transformations of the bulk equations of motion and showed that for spins larger than two there is an anomaly: no local boundary term can reproduce all the gauge symmetry of the bulk. Next, we have written the solution of the bulk equations of motion in terms of a boundary higher-spin conformal field $h^{(s)}$, that is a $d$-dimensional field subject to gauge and HS Weyl transformations. Using these two building blocks, the boundary action and the boundary field, we determined the on-shell action $S_{z_{B}}\left[h^{(s)}\right]$.

The key motivation of our investigation is the HS/CFT correspondence. Let us examine how the on-shell action is described from the boundary CFT point of view. The boundary CFT is the theory of $N$ free massless complex scalars, $\phi=\left(\phi^{1}, \cdots, \phi^{N}\right)$, in $d$ dimensions. The operators dual to the AdS HS gauge fields are the currents of higher ranks:

$$
\bar{J}^{(s) \mu_{1} \cdots \mu_{s}}=\sum_{r=0}^{s} c_{r, s} \partial^{\mu_{1}} \cdots \partial^{\mu_{r}} \boldsymbol{\phi}^{*} \cdot \partial^{\mu_{r+1}} \cdots \partial^{\mu_{s}} \boldsymbol{\phi},
$$

where the coefficients $c_{r, s}$ 's are chosen such that these currents are conserved and traceless when the scalar is on shell:

$$
\partial_{\mu_{1}} \bar{J}^{(s) \mu_{1} \cdots \mu_{s}} \approx 0, \quad \eta_{\mu_{1} \mu_{2}} \bar{J}^{(s) \mu_{1} \cdots \mu_{s}} \approx 0 .
$$

The connected correlation functions of these currents can be generated by the effective action $W_{\Lambda}\left[\tilde{h}^{(0)}, \tilde{h}^{(1)}, \cdots\right]$ given by

$$
e^{-W_{\Lambda}}:=\int_{\Lambda} \mathcal{D} \boldsymbol{\phi} \exp \left(-\int d^{d} x \partial_{\mu} \boldsymbol{\phi}^{*} \cdot \partial^{\mu} \boldsymbol{\phi}+\sum_{s=0}^{\infty}\left\langle\left\langle\tilde{h}^{(s)} \mid \bar{J}^{(s)}\right\rangle\right),\right.
$$

where the (boundary) HS fields $\tilde{h}^{(s)}$ is the source for the currents $\bar{J}^{(s)}$, and $\Lambda$ is the UV regularization parameter of mass dimension. The effective action $W_{\Lambda}$ (5.3) is a functional of the conformal HS fields $\tilde{h}^{(s)}$. According to the HS/CFT ccorrespondence the finite part of $W_{\Lambda}$ is given, in the semi-classical regime, by the finite part of the bulk on-shell action. The traceless-ness and transversality of the currents (5.2) induce respectively the diffeomorphism-like and the Weyl-transformation-like gauge transformations on $\tilde{h}^{(s)}$ :

$$
\delta \tilde{h}^{(s)}=u \cdot \partial \lambda^{(s-1)}-u^{2} \sigma^{(s-2)}+\mathcal{O}(\tilde{h}, \lambda)+\mathcal{O}(\tilde{h}, \sigma),
$$

where $\mathcal{O}(\tilde{h}, \lambda)$ and $\mathcal{O}(\tilde{h}, \sigma)$ are linear in $\tilde{h}^{\left(s^{\prime}\right)}, \lambda^{(r)}$ and $\sigma^{\left(r^{\prime}\right)}$ for any $s^{\prime}, r, r^{\prime}$, and their explicit expressions are given in [45].

When $\tilde{h}^{(s)}$ is restricted to only spin 2 field, the transformations (5.4) reduce to the diffeomorphism and the Weyl transformation. Here, it is important to note that the gauge field $\tilde{h}$ and the gauge parameters $\lambda$ and $\sigma$ are not subject to any trace constraints, and also 
that the deformations of the linear gauge transformations are exact in the first order of $\tilde{h}$ : there is no $\mathcal{O}\left(\tilde{h}^{2}\right)$ term. In [45], it has been shown that $W_{\Lambda}$ is invariant only under the $\lambda$-transformations while the $\sigma$-transformations are anomalous: $\delta_{\lambda} W_{\Lambda}=0$ and $\delta_{\sigma} W_{\Lambda} \neq 0$. Further analysis was performed by expanding $W_{\Lambda}$ in $\Lambda$ as

$$
W_{\Lambda}=\sum_{n=-\infty}^{[d / 2]} \Lambda^{-2 n+d} W_{n}+W_{\text {fin }}-\log \frac{\Lambda}{\mu} W_{\log }+o\left(\Lambda^{-1}\right),
$$

and it has been shown that

$$
\delta_{\sigma} W_{\mathrm{fin}}=0 \quad[d: \text { odd }], \quad \delta_{\sigma} W_{\log }=0 \quad[d: \text { even }],
$$

which generalizes the case of the spin 2 Weyl anomaly. The explicit expressions of $W_{\text {fin }}$ and $W_{\log }$ were determined perturbatively in the fields in ref. [45], the quadratic terms can be expressed in terms of the on-shell action as

$$
\begin{array}{ll}
-W_{\text {fin }}[\tilde{h}]=\sum_{s=0}^{\infty} S_{\text {fin }}\left[C_{s, d} \tilde{h}^{(s)}\right]+\mathcal{O}\left(\tilde{h}^{3}\right) & {[d: \text { odd }],} \\
-W_{\log }[\tilde{h}]=\sum_{s=0}^{\infty} S_{\log }\left[C_{s, d} \tilde{h}^{(s)}\right]+\mathcal{O}\left(\tilde{h}^{3}\right) & {[d: \text { even }],}
\end{array}
$$

with

$$
C_{s, d}^{2}=\frac{\Gamma^{2}\left(\frac{d+2 s-4}{2}\right)}{\pi^{\frac{d-1}{2}} 2^{d+s+1} \Gamma\left(\frac{d+2 s-1}{2}\right)} .
$$

Hence, the quadratic parts of the CFT effective action coincide with the on-shell actions $S_{\text {fin }}$ and $S_{\log }(4.42,4.43)$ up to field redefinitions with multiplicative constants. The same is also true for the non-local part of $S_{\text {fin }}$ for even $d$.

In this paper, we have shown that the AdS counterpart of the HS Weyl anomaly is due essentially to the fact that the boundary term, which is necessary to obtain the classical HS EOM, cannot be made invariant under the whole bulk gauge group. More precisely, the onshell action breaks the symmetries generated by the gauge parameters $\epsilon^{(s-2)}$ or $\varepsilon_{d \mu_{1} \cdots \mu_{s-2}}$. At the quadratic level, the finite part of the on-shell action is anomalous only for an even dimensional boundary and in this case the anomaly is due to local terms, thus contributing with only contact terms to the two-point correlation function. More precisely, the non-local part of the on-shell action is anomaly-free for any dimension and coincides with that of the effective action determined in [45]. Consequently, the correlation function of two currents $\left\langle\bar{J}_{\mu_{1} \cdots \mu_{s}}(x) \bar{J}_{\nu_{1} \cdots \nu_{s}}\left(x^{\prime}\right)\right\rangle$ obtained from the on-shell action by functional differentiation with respect to the conformal HS fields coincides with the one obtained from the free boundary CFT up to possible contact terms. The way to extract the correlation functions from the effective action has been shown in [45]. The anomaly is expected to play a more important role when considering higher orders since in the CFT side the generalized Weyl anomalies arise starting from cubic orders (see $[55,56]$ for recent constructions of cubic interaction vertices of AdS HS fields). A key problem for the future is to gain a better understanding of the HS interactions in the metric form from the CFT effective action. 
Another important problem is to see via the AdS/CFT correspondence how the nonAbelian deformation of the gauge transformation is realized in the bulk of AdS. In fact, it was shown in [57] that the collection of all the HS fields can be grouped in a Hermitian operator acting on the scalar field and that the transformations (5.4) arise from the leading terms of respectively the Hermitian and anti-Hermitian transformations acting on the scalar fields. The symmetry group of the CFT effective action is thus generated by the algebra of Hermitian operators. An important issue is the bulk counterpart of this symmetry group. A step in this direction was proposed by the correspondence which we made explicit between the boundary conformal HS field and the bulk HS field. We hope to come back to this issue in the future.

\section{Acknowledgments}

We are grateful to X. Bekaert and A. Sagnotti for many helpful discussions, and to APCParis VII and Scuola Normale Superiore for the kind hospitality extended to one or more of us. The present research was supported in part by Scuola Normale Superiore, by INFN and by the MIUR-PRIN contract 2009-KHZKRX.

\section{A Linearized AdS Gravity}

We linearize the action for the AdS Gravity. We first redefine the metric tensor as

$$
G_{M N}(x, z)=\frac{\eta_{M N}+\kappa_{M N}(x, z)}{z^{2}},
$$

and then expand the Hilbert-Einstein (HE) action $\mathcal{I}_{H E}$ up to quadratic order in $\kappa_{M N}$. The latter is related to the notation used in this paper as

$$
\varphi(x, z ; U)=\frac{1}{2} U^{M} U^{N} \kappa_{M N}(x, z) .
$$

We perform integrations by part to express $\mathcal{I}_{H E}$ as

$$
\mathcal{S}_{H E}[G]=\mathcal{I}_{\mathcal{M}}[\kappa]+\mathcal{I}_{\partial \mathcal{M}}^{H E}[\kappa]+\mathcal{O}\left(\kappa^{3}\right),
$$

where $\mathcal{I}_{\mathcal{M}}$ is the bulk action whose Lagrangian is proportional to the EOM and $\mathcal{I}_{\partial \mathcal{M}}^{H E}$ the resulting boundary term. Explicitly the bulk action is given by

$$
\mathcal{I}_{\mathcal{M}}[\kappa]=-\frac{1}{4} \int_{z_{B}}^{\infty} \frac{d z}{z^{d-1}} \int d^{d} x\left(\kappa^{M N} F_{M N}-\frac{1}{2} \kappa_{M}^{M} F_{N}^{N}\right),
$$

where the indices $M, N$ are raised by the flat metric $\eta^{M N}$ and the tensor $F_{M N}$ is the linearization of $-2\left(R_{M N}+d G_{M N}\right)$. The remaining part of (A.3) is the boundary term $\mathcal{I}_{\partial \mathcal{M}}^{H E}$, and it is given by

$$
\begin{aligned}
\mathcal{I}_{\partial \mathcal{M}}^{H E}[\kappa]=\int \frac{d^{d} x}{z_{B}^{d}}[ & -z \partial_{z}\left(\kappa_{\mu}^{\mu}-\frac{3}{8} \kappa_{\mu \nu}^{2}+\frac{1}{8}\left(\kappa_{\mu}^{\mu}\right)^{2}\right)+2\left(1+\frac{1}{2} \kappa_{\mu}^{\mu}-\frac{1}{4} \kappa_{\mu \nu}^{2}+\frac{1}{8}\left(\kappa_{\mu}^{\mu}\right)^{2}\right) \\
& +\frac{1}{2} \kappa_{d d} z \partial_{z} \kappa_{\mu}^{\mu}+\frac{1}{2} z\left(\kappa^{\mu \nu} \partial_{\mu} \kappa_{\nu d}+2 \kappa^{\mu d} \partial_{\mu} \kappa_{d d}+\kappa^{\mu d} \partial_{\mu} \kappa_{\nu}^{\nu}\right) \\
& \left.-d\left(\kappa_{d d}-\kappa_{\mu d}^{2}-\frac{3}{4} \kappa_{d d}^{2}\right)-\frac{1}{4}(d+1) \kappa_{d d} \kappa_{\nu}^{\nu}\right]_{z=z_{B}}
\end{aligned}
$$


The HE action should be complemented by the York-Gibbons-Hawking (GH) term. We linearize the latter as $\mathcal{S}_{G H}[G]=\mathcal{I}_{\partial \mathcal{M}}^{G H}[\kappa]+\mathcal{O}\left(\kappa^{3}\right)$ with

$$
\begin{aligned}
\mathcal{I}_{\partial \mathcal{M}}^{G H}[\kappa]=\int \frac{d^{d} x}{z_{B}^{d}}[ & z \partial_{z}\left(\kappa_{\mu}^{\mu}-\frac{1}{2} \kappa_{\mu \nu}^{2}+\frac{1}{4}\left(\kappa_{\mu}^{\mu}\right)^{2}\right)-2 d\left(1+\frac{1}{2} \kappa_{\mu}^{\mu}-\frac{1}{4} \kappa_{\mu \nu}^{2}+\frac{1}{8}\left(\kappa_{\mu}^{\mu}\right)^{2}\right) \\
& \left.-\frac{1}{2} \kappa_{d d} z \partial_{z} \kappa_{\mu}^{\mu}+\kappa_{d d} z \partial^{\mu} \kappa_{\mu d}+d\left(\kappa_{d d}-\kappa_{\mu d}^{2}-\frac{3}{4} \kappa_{d d}^{2}+\frac{1}{2} \kappa_{d d} \kappa_{\mu}^{\mu}\right)\right]_{z=z_{B}} .
\end{aligned}
$$

Besides the GH term, we may include an additional boundary term proportional to the boundary volume $V_{\partial \mathcal{M}}[\gamma]=\mathcal{I}_{\partial \mathcal{M}}^{V}[\kappa]+\mathcal{O}\left(\kappa^{3}\right)$ :

$$
\mathcal{I}_{\partial \mathcal{M}}^{V}[\kappa]=\int \frac{d^{d} x}{z_{B}^{d}}\left[1+\frac{1}{2} \kappa_{\mu}^{\mu}-\frac{1}{4} \kappa_{\mu \nu}^{2}+\frac{1}{8}\left(\kappa_{\mu}^{\mu}\right)^{2}\right]_{z=z_{B}} .
$$

Finally we consider the following action for AdS Gravity:

$$
\mathcal{S}_{H E}[G]+\mathcal{S}_{G H}[G]+c V_{\partial \mathcal{M}}[\gamma]=\mathcal{I}_{\mathcal{M}}[\kappa]+\mathcal{I}_{\partial \mathcal{M}}[\kappa]+\mathcal{O}\left(\kappa^{3}\right)
$$

where $\mathcal{I}_{\partial \mathcal{M}}$ is the sum of at most quadratic part of different boundary terms:

$$
\begin{aligned}
& \mathcal{I}_{\partial \mathcal{M}}[\kappa]=\mathcal{I}_{\partial \mathcal{M}}^{H E}[\kappa]+\mathcal{I}_{\partial \mathcal{M}}^{G H}[\kappa]+c \mathcal{I}_{\partial \mathcal{M}}^{V}[\kappa] \\
& =\int \frac{d^{d} x}{z_{B}^{d}}\left[-\frac{1}{8} z \partial_{z}\left(\kappa_{\mu \nu}^{2}-\left(\kappa_{\mu}^{\mu}\right)^{2}\right)+[c-2(d-1)]\left(1+\frac{1}{2} \kappa_{\mu}^{\mu}-\frac{1}{4} \kappa_{\mu \nu}^{2}+\frac{1}{8}\left(\kappa_{\mu}^{\mu}\right)^{2}\right)\right. \\
& \left.\quad+\frac{1}{2} \kappa^{\mu \nu} z \partial_{\mu} \kappa_{\nu d}-\frac{1}{2} \kappa_{\mu}^{\mu} z \partial^{\nu} \kappa_{\nu d}+\frac{1}{4}(d-1) \kappa_{\mu}^{\mu} \kappa_{d d}\right]_{z=z_{B}} .
\end{aligned}
$$

The choice $c=2(d-1)$ gives us a particularly simple result where the constant term as well as the linear term in $\kappa$ disappear.

\section{B Radial decomposition of the Fronsdal operator}

For an explicit analysis of the Fronsdal theory, it is useful to decompose the Fronsdal operator as follows:

$$
\mathcal{F}=\sum_{n=0}^{2} \sum_{m=0}^{4} v^{n} \mathcal{F}_{m}^{n} \partial_{v}^{m},
$$

where $\mathcal{F}_{m}^{n}$ are operators without $v$ and $\partial_{v}$ given by

$$
\begin{aligned}
\mathcal{F}_{0}^{0} & =\left(z \partial_{z}\right)^{2}-d z \partial_{z}-m_{s}^{2}-s+z^{2} \mathcal{F}_{M^{d}}-\frac{1}{2} u^{2}\left(z \partial_{z}+u \cdot \partial_{u}\right) \partial_{u}^{2}, \\
\mathcal{F}_{1}^{0} & =z u^{2}\left(\partial \cdot \partial_{u}\right)+z(u \cdot \partial)\left(d-2-z \partial_{z}+u \cdot \partial_{u}-u^{2} \partial_{u}^{2}\right), \\
\mathcal{F}_{2}^{0} & =\frac{1}{2}\left[z^{2}(u \cdot \partial)^{2}+u^{2}\left(z \partial_{z}-2 d-3 u \cdot \partial_{u}+u^{2} \partial_{u}^{2}\right)\right], \\
\mathcal{F}_{3}^{0} & =-z u^{2}(u \cdot \partial), \quad \mathcal{F}_{4}^{0}=\frac{1}{2}\left(u^{2}\right)^{2}, \\
\mathcal{F}_{0}^{1} & =z\left(u \cdot \partial \partial_{u}^{2}-\partial \cdot \partial_{u}\right)\left(z \partial_{z}+u \cdot \partial_{u}-2\right)
\end{aligned}
$$




$$
\begin{aligned}
& \mathcal{F}_{1}^{1}=-d-2 s+3+\left(z \partial_{z}+u \cdot \partial_{u}\right)\left(d-z \partial_{z}+u \cdot \partial_{u}\right)-u^{2}\left(z \partial_{z}+u \cdot \partial_{u}\right) \partial_{u}^{2}, \\
& \mathcal{F}_{2}^{1}=z(u \cdot \partial)\left(z \partial_{z}+u \cdot \partial_{u}+1\right), \\
& \mathcal{F}_{3}^{1}=-u^{2}\left(z \partial_{z}+u \cdot \partial_{u}+1\right), \quad \mathcal{F}_{4}^{1}=0, \\
& \mathcal{F}_{0}^{2}=\frac{1}{2}\left(z \partial_{z}+u \cdot \partial_{u}\right)\left(z \partial_{z}+u \cdot \partial_{u}-2\right) \partial_{u}^{2}, \\
& \mathcal{F}_{1}^{2}=0, \quad \mathcal{F}_{2}^{2}=1+\frac{1}{2}\left(z \partial_{z}+u \cdot \partial_{u}\right)^{2}, \quad \quad \mathcal{F}_{3}^{2}=\mathcal{F}_{4}^{2}=0 .
\end{aligned}
$$

In (2.52), the gauge variation of the bulk Fronsdal action is given by two quantities: $\left.\partial_{v} \mathcal{F} \varphi^{(s)}\right|_{v=0}$ and $\left.\left(\partial_{u}^{2}-\partial_{v}^{2}\right) \mathcal{F} \varphi^{(s)}\right|_{v=0}$. Here, we compute them explicitly. First we rewrite them in terms of the decomposed operators $\mathcal{F}_{m}^{n}$ and then use the results of (B.2). Finally the explicit expressions for these two quantities are

$$
\begin{aligned}
\left(\partial_{v}\right. & \left.\mathcal{F} \varphi^{(s)}\right)(x, z ; u, 0)= \\
= & {\left[\mathcal{F}_{0}^{1}-\mathcal{F}_{3}^{0}\left(\partial_{u}^{2}\right)^{2}\right] \phi^{(s)}(x, z ; u)+\left[\mathcal{F}_{0}^{0}+\mathcal{F}_{1}^{1}-\mathcal{F}_{4}^{0}\left(\partial_{u}^{2}\right)^{2}\right] \phi^{(s-1)}(x, z ; u) } \\
& +\left[\mathcal{F}_{1}^{0}+\mathcal{F}_{2}^{1}-2 \mathcal{F}_{3}^{0} \partial_{u}^{2}\right] \phi^{(s-2)}(x, z ; u)+\left[\mathcal{F}_{2}^{0}+\mathcal{F}_{3}^{1}-2 \mathcal{F}_{4}^{0} \partial_{u}^{2}\right] \phi^{(s-3)}(x, z ; u) \\
= & {\left[z\left(u \cdot \partial \partial_{u}^{2}-\partial \cdot \partial_{u}\right)\left(z \partial_{z}+s-2\right)+z u \cdot \partial u^{2}\left(\partial_{u}^{2}\right)^{2}\right] \phi^{(s)}(x, z ; u) } \\
& +\left[z^{2} \mathcal{F}_{M^{d}}-\frac{3}{2} u^{2}\left(z \partial_{z}+s-3\right) \partial_{u}^{2}-\frac{1}{2}\left(u^{2}\right)^{2}\left(\partial_{u}^{2}\right)^{2}\right] \phi^{(s-1)}(x, z ; u) \\
& +\left[(d+2 s-5) z u \cdot \partial+z u^{2}\left(\partial \cdot \partial_{u}+u \cdot \partial \partial_{u}^{2}\right)\right] \phi^{(s-2)}(x, z ; u) \\
& +\left[\frac{1}{2} z^{2}(u \cdot \partial)^{2}-\frac{1}{2} u^{2}\left(z \partial_{z}+2 d+5 s-13+u^{2} \partial_{u}^{2}\right)\right] \phi^{(s-3)}(x, z ; u),
\end{aligned}
$$

and

$$
\begin{aligned}
& \left(\left(\partial_{u}^{2}-\partial_{v}^{2}\right) \mathcal{F} \varphi^{(s)}\right)(x, z ; u, 0)= \\
& =\left[\partial_{u}^{2}\left(\mathcal{F}_{0}^{0}-\mathcal{F}_{4}^{0}\left(\partial_{u}^{2}\right)^{2}\right)-2 \mathcal{F}_{0}^{2}+\left(\mathcal{F}_{2}^{0}+2 \mathcal{F}_{3}^{1}-2 \mathcal{F}_{4}^{0} \partial_{u}^{2}\right)\left(\partial_{u}^{2}\right)^{2}\right] \phi^{(s)}(x, z ; u) \\
& \quad+\left[\partial_{u}^{2} \mathcal{F}_{1}^{0}-2 \mathcal{F}_{0}^{1}+\mathcal{F}_{3}^{0}\left(\partial_{u}^{2}\right)^{2}\right] \phi^{(s-1)}(x, z ; u) \\
& \quad+\left[\partial_{u}^{2}\left(\mathcal{F}_{2}^{0}-2 \mathcal{F}_{4}^{0} \partial_{u}^{2}\right)-\mathcal{F}_{0}^{0}-2 \mathcal{F}_{1}^{1}-2 \mathcal{F}_{2}^{2}+\left(2 \mathcal{F}_{2}^{0}+4 \mathcal{F}_{3}^{1}-3 \mathcal{F}_{4}^{0} \partial_{u}^{2}\right) \partial_{u}^{2}\right] \phi^{(s-2)}(x, z ; u) \\
& \quad+\left[\partial_{u}^{2} \mathcal{F}_{3}^{0}-\mathcal{F}_{1}^{0}-2 \mathcal{F}_{2}^{1}+2 \mathcal{F}_{3}^{0} \partial_{u}^{2}\right] \phi^{(s-3)}(x, z ; u) \\
& =\left[-2(d+2 s-5)\left(z \partial_{z}+s-2\right) \partial_{u}^{2}-u^{2}\left(2 z \partial_{z}+3 d+8 s-25+u^{2} \partial_{u}^{2}\right)\left(\partial_{u}^{2}\right)^{2}\right. \\
& \left.\quad+z^{2}\left[2\left(\square \partial_{u}^{2}-\left(\partial_{u} \cdot \partial\right)^{2}\right)+u \cdot \partial\left(\partial_{u} \cdot \partial+u \cdot \partial \partial_{u}^{2}\right) \partial_{u}^{2}\right]\right] \phi^{(s)}(x, z ; u) \\
& \quad+z\left[4(d+2 s-5) \partial_{u} \cdot \partial-\left(3 z \partial_{z}+d+5 s-11\right) u \cdot \partial \partial_{u}^{2}\right. \\
& \left.\quad-u^{2} \partial_{u} \cdot \partial \partial_{u}^{2}-2 u^{2} u \cdot \partial\left(\partial_{u}^{2}\right)^{2}\right] \phi^{(s-1)}(x, z ; u) \\
& +\left[z^{2} u \cdot \partial\left(3 \partial_{u} \cdot \partial+u \cdot \partial \partial_{u}^{2}\right)-\left(u^{2}\right)^{2}\left(\partial_{u}^{2}\right)^{2}\right. \\
& \left.\quad-(5 d+10 s-29) u^{2} \partial_{u}^{2}-2(d+2 s-4)(d+2 s-5)\right] \phi^{(s-2)}(x, z ; u) \\
& -z\left[u \cdot \partial\left(z \partial_{z}+3 d+7 s-17+2 u^{2} \partial_{u}^{2}\right)+3 u^{2} \partial_{u} \cdot \partial\right] \phi^{(s-3)}(x, z ; u) .
\end{aligned}
$$




\section{Useful identities}

In this section, we summarize the mathematical identities that has been used in the paper. First, the P-operators introduced for the construction of boundary actions satisfy

$$
\begin{aligned}
& \mathrm{P}_{\mathrm{e}}-\mathrm{P}_{\mathrm{o}}=u^{2} \mathrm{P}_{\mathrm{o}}^{\prime} \partial_{u}^{2}, \quad \mathrm{P}_{\mathrm{e}}+\mathrm{P}_{\mathrm{e}}^{\prime}=u^{2} \mathrm{P}_{\mathrm{o}} \partial_{u}^{2}, \\
& {\left[3\left(d+2 u \cdot \partial_{u}+1\right)+u^{2} \partial_{u}^{2}\right] \mathrm{P}_{\mathrm{o}}^{\prime}=\left(d+2 u \cdot \partial_{u}+1\right) \mathrm{P}_{\mathrm{o}},}
\end{aligned}
$$

and

$$
\begin{array}{ll}
\partial_{u} \cdot \partial \mathrm{P}_{\mathrm{e}}^{\prime}=\mathrm{P}_{\mathrm{e}}\left(u \cdot \partial \partial_{u}^{2}-\partial_{u} \cdot \partial\right), & \partial_{u}^{2} \mathrm{P}_{\mathrm{e}}^{\prime}=\left(d+2 u \cdot \partial_{u}-1\right) \mathrm{P}_{\mathrm{e}} \partial_{u}^{2}, \\
\partial_{u} \cdot \partial \mathrm{P}_{\mathrm{e}}=\mathrm{P}_{\mathrm{o}}\left(u \cdot \partial \partial_{u}^{2}+\partial_{u} \cdot \partial\right), & \partial_{u}^{2} \mathrm{P}_{\mathrm{e}}=\left(d+2 u \cdot \partial_{u}+1\right) \mathrm{P}_{\mathrm{o}} \partial_{u}^{2}, \\
\partial_{u} \cdot \partial \mathrm{P}_{\mathrm{o}}=\mathrm{P}_{\mathrm{o}}^{\prime}\left(u \cdot \partial \partial_{u}^{2}+3 \partial_{u} \cdot \partial\right), & \partial_{u}^{2} \mathrm{P}_{\mathrm{o}}=\left(d+2 u \cdot \partial_{u}+3\right) \mathrm{P}_{\mathrm{o}}^{\prime} \partial_{u}^{2}
\end{array}
$$

The Bessel-like function $U_{\nu}(z)$ appears in the solutions of the Fronsdal equation. It is a related to the modified Bessel function of second kind:

$$
U_{\nu}(z):=\frac{2}{\Gamma(\nu)}\left(\frac{z}{2}\right)^{\nu} K_{\nu}(z)=1+\mathcal{O}\left(z^{2}\right),
$$

and it satisfies the following Bessel-like differential equation:

$$
\left[\left(z \partial_{z}-\nu\right)^{2}-a z^{2}-\nu^{2}\right] U_{\nu}(a z)=0 .
$$

In order to study the finite part or the logarithmically divergent part of the on-shell action, we need the series expansion of this function:

$$
U_{\nu}(z)=\sum_{k=0}^{n_{\nu}} \frac{\left(\frac{z}{2}\right)^{2 k}}{(1-\nu)_{k} k !}+\frac{\left(\frac{z}{2}\right)^{2 \nu}}{\Gamma(\nu) \Gamma(\nu+1)} \sum_{k=0}^{\infty} u_{\nu, k}(z) \frac{\left(\frac{z}{2}\right)^{2 k}}{(1+\nu)_{k} k !},
$$

where $n_{\nu}$ is given by

$$
n_{\nu}=\left\{\begin{array}{cc}
\infty & {\left[\nu-\frac{1}{2} \in \mathbb{N}\right]} \\
\nu-1 & {[\nu \in \mathbb{N}]}
\end{array}\right.
$$

and $u_{\nu, k}(z)$ by

$$
u_{\nu, k}(z)=\left\{\begin{array}{cc}
\pi(-1)^{\nu+\frac{1}{2}} & {\left[\nu-\frac{1}{2} \in \mathbb{N}\right]} \\
(-1)^{\nu}\left[\psi(k+1)+\psi(k+\nu+1)-2 \ln \left(\frac{z}{2}\right)\right] & {[\nu \in \mathbb{N}]}
\end{array} .\right.
$$

The function $U_{\nu}(z)$ enjoys several identities that can be proven from the identities of the modified Bessel function $K_{\nu}$. For example, it satisfies the following recurrence identity:

$$
U_{\nu}(z)-U_{\nu-1}(z)=\frac{z^{2}}{4(\nu-1)(\nu-2)} U_{\nu-2}(z) .
$$

The derivatives of $U_{\nu}$ can be again expressed in terms of $U_{\nu^{\prime}}$ with a neighboring index:

$$
z \partial_{z} U_{\nu}(z)=-\frac{z^{2}}{2(\nu-1)} U_{\nu-1}(z) .
$$




\section{Gauge variation of the bulk action and the boundary action}

The variation of the bulk action under $\epsilon^{(s-2)}$ gives

$$
\delta_{\epsilon^{(s-2)}} \mathcal{I}_{\mathcal{M}}=\frac{z_{B}^{-d}}{2}\left\langle\epsilon^{(s-2)} \mid \mathrm{P}_{\mathrm{o}} \frac{1}{2}\left(\partial_{v}^{2}-\partial_{u}^{2}\right) \mathcal{F} \varphi\right\rangle_{z_{B}}=\frac{z_{B}^{-d}}{2}\left\langle\left\langle\epsilon^{(s-2)} \mid J_{\mathcal{M}}\right\rangle_{z_{B}},\right.
$$

where $J_{\mathcal{M}}$ is given by

$$
\begin{aligned}
J_{\mathcal{M}}=\mathrm{P}_{\mathrm{o}}[ & \left(d+2 s-5+u^{2} \partial_{u}^{2}\right) \partial_{u}^{2} \chi^{(s)}+z u \cdot \partial \chi^{(s-3)}+\left(d+2 s-4+u^{2} \partial_{u}^{2}\right) \zeta^{(s-2)} \\
& -\frac{1}{2} z^{2}\left(\partial_{u}^{2} B^{(s)}-B^{(s-2)}\right)-\frac{1}{2}\left(3 d+6 s-11+3 u^{2} \partial_{u}^{2}\right) u^{2} \partial_{u}^{2} \phi^{(s)} \\
& \left.+\frac{1}{2}\left(d+2 s-1+u^{2} \partial_{u}^{2}\right) u^{2} \partial_{u}^{2} \phi^{(s-2)}+z u^{2} \partial_{u} \cdot \partial \psi^{(s-3)}\right]
\end{aligned}
$$

where we used the gauge invariants:

$$
\begin{aligned}
B^{(s)} & :=\mathcal{F}_{M^{d}} \phi^{(s)}+\frac{1}{2}(u \cdot \partial)^{2} \phi^{(s-2)}, \\
B^{(s-2)} & :=\mathcal{F}_{M^{d}} \phi^{(s-2)}-\frac{1}{2}(u \cdot \partial)^{2}\left[\left(\partial_{u}^{2}\right)^{2} \phi^{(s)}+2 \partial_{u}^{2} \phi^{(s-2)}\right] .
\end{aligned}
$$

The variation of the boundary terms $(2.69)$ under $\epsilon^{(s-2)}$ gives

$$
\delta_{\epsilon^{(s-2)}} \mathcal{I}_{\partial \mathcal{M}}=\frac{z_{B}^{-d}}{2}\left\langle\left\langle\epsilon^{(s-2)} \mid J_{\partial \mathcal{M}}\right\rangle_{z_{B}},\right.
$$

where $J_{\partial \mathcal{M}}$ is given by

$$
\begin{aligned}
J_{\partial \mathcal{M}}=\mathrm{P}_{\mathrm{o}}[ & -\left(d+2 s-5+u^{2} \partial_{u}^{2}\right) \partial_{u}^{2} \chi^{(s)}-z u \cdot \partial \chi^{(s-3)}+(d+s-3) z u \cdot \partial \psi^{(s-3)} \\
& +z^{2} u \cdot \partial\left(u \cdot \partial \partial_{u}^{2}+3 \partial_{u} \cdot \partial\right)\left(\xi^{(s-2)}+\mathrm{T} \zeta^{(s-2)}\right) \\
& -z^{2}\left[\square \partial_{u}^{2}-\left(\partial_{u} \cdot \partial\right)^{2}+2 u \cdot \partial \partial_{u} \cdot \partial \partial_{u}^{2}+(u \cdot \partial)^{2}\left(\partial_{u}^{2}\right)^{2}\right] \phi^{(s)} \\
& -\left(s-2+\frac{1}{2} \partial_{u}^{2} u^{2}+\frac{1}{2} u^{2} \partial_{u}^{2}\right) \zeta^{(s-2)} \\
& \left.+\left[\left(2(s-2)+u^{2} \partial_{u}^{2}\right)\left(3(d+2 s-5)+u^{2} \partial_{u}^{2}\right)-2 \partial_{u}^{2}\left(u^{2}\right)^{2} \partial_{u}^{2}\right]\left(\xi^{(s-2)}+\mathrm{T} \zeta^{(s-2)}\right)\right] \\
-(d & +2 s-5)\left[\left(3(s-2)+u^{2} \partial_{u}^{2}\right) \mathrm{P}_{\mathrm{e}} \partial_{u}^{2} \phi^{(s)}+z u \cdot \partial \mathrm{P}_{\mathrm{o}} \psi^{(s-3)}\right] .
\end{aligned}
$$

\section{E Counterterms}

The $\epsilon^{(s-2)}$-variations of the counterterm $\mathcal{I}_{\partial \mathcal{M}}^{2 \text { (c.t.) }}(3.8)$ can be obtained by noticing first that it depends on $\beta^{(s-3)}$ through $\gamma^{(s-2)}$

$$
\gamma^{(s-2)}=z u \cdot \partial \beta^{(s-3)},
$$

so that

$$
\begin{aligned}
\frac{z_{B}^{d}}{c} \mathcal{I}_{\partial \mathcal{M}}^{2(\text { c.t. })}= & \left\langle\left\langle\phi^{(s)} \mid G^{(s)}\right\rangle_{z_{B}}-\left\langle\left\langle\gamma^{(s-2)} \mid A^{(s-2)}\right\rangle_{z_{B}}\right.\right. \\
= & \left\langle\left\langle\phi^{(s)} \mid \mathcal{G}_{\phi \phi} \phi^{(s)}\right\rangle_{z_{B}}+\left\langle\left\langle\phi^{(s)} \mid \mathcal{G}_{\phi \gamma} \gamma^{(s-2)}\right\rangle_{z_{B}}\right.\right. \\
& +\left\langle\left\langle\gamma^{(s-2)} \mid \mathcal{G}_{\gamma \phi} \phi^{(s)}\right\rangle_{z_{B}}+\left\langle\left\langle\gamma^{(s-2)} \mid \mathcal{G}_{\gamma \gamma} \gamma^{(s-2)}\right\rangle_{z_{B}},\right.\right.
\end{aligned}
$$


with

$$
\begin{aligned}
& \mathcal{G}_{\phi \phi}=z^{2}\left[\square-u \cdot \partial \partial_{u} \cdot \partial+\frac{1}{2}(u \cdot \partial)^{2} \partial_{u}^{2}-\frac{1}{2} u^{2}\left(\square \partial_{u}^{2}-\left(\partial_{u} \cdot \partial\right)^{2}-\frac{1}{2} u \cdot \partial \partial_{u} \cdot \partial \partial_{u}^{2}\right)\right], \\
& \mathcal{G}_{\phi \gamma}=\frac{z^{2}}{2}\left[u^{2}\left(\square+\frac{1}{2} u \cdot \partial \partial_{u} \cdot \partial\right)-(u \cdot \partial)^{2}\right], \\
& \mathcal{G}_{\gamma \phi}=\mathcal{G}_{\phi \gamma}^{\dagger}, \quad \mathcal{G}_{\gamma \gamma}=z^{2}\left(\square+\frac{1}{2} u \cdot \partial \partial_{u} \cdot \partial\right) .
\end{aligned}
$$

Notice the selfadjoint-ness of the above operators:

$$
\mathcal{G}_{i j}^{\dagger}=\mathcal{G}_{j i},
$$

so that under an arbitrary variation, we have

$$
\delta \mathcal{I}_{\partial \mathcal{M}}^{2 \text { (c.t.) }}=2 c z_{B}^{-d}\left[\left\langle\delta \delta \phi^{(s)} \mid G^{(s-3)}\right\rangle_{z_{B}}+\left\langle\left\langle\delta \beta^{(s-3)} \mid K^{(s-3)}\right\rangle_{z_{B}}\right] .\right.
$$

Using the $\epsilon^{(s-2)}$-variations:

$$
\delta_{\epsilon^{(s-2)}} \phi^{(s)}=-u^{2} \epsilon^{(s-2)}, \quad \delta_{\epsilon^{(s-2)}} \beta^{(s-3)}=z \mathrm{~T}\left(u \cdot \partial \partial_{u}^{2}+3 \partial_{u} \cdot \partial\right) \epsilon^{(s-2)},
$$

we obtain (E.5).

\section{F Traceless and transverse projection}

The traceless and transverse part of field $h^{(s)}$ can be uniquely determined from the gauge transformation:

$$
\delta h^{(s)}=u \cdot \partial \lambda^{(s-1)}-u^{2} \sigma^{(s-2)} .
$$

Without loss of generality, we assume that the gauge parameter $\lambda^{(s-1)}$ is traceless: $\lambda^{(s-1)}=$ $\bar{\lambda}^{(s-1)}$, since its trace part overlaps the divergent part of $\lambda^{(s-2)}$. Then, the trace of $\sigma^{(s-2)}$ is uniquely fixed by the double trace part of $h^{(s)}$ via

$$
\left(\partial_{u}^{2}\right)^{2} h^{(s)}+\left[4(d+2 s-6)+u^{2} \partial_{u}^{2}\right] \partial_{u}^{2} \sigma^{(s-2)}=0 .
$$

Then, we have a doubly traceless field $\overline{\bar{h}}^{(s)}$ with two traceless parameters $\bar{\lambda}^{(s-1)}$ and $\bar{\sigma}^{(s-2)}$. Now, we use the equations of the traceless and transverse gauge conditions. First, the traceless gauge condition determines $\bar{\sigma}^{(s-2)}$ in terms of $\overline{\bar{h}}^{(s)}$ and $\bar{\lambda}^{(s-1)}$ as

$$
\bar{\sigma}^{(s-2)}=-\frac{1}{2(d+2 s-4)}\left[2 \partial_{u} \cdot \partial \bar{\lambda}^{(s-1)}+\partial_{u}^{2} \overline{\bar{h}}^{(s)}\right] .
$$

By plugging in this solution into the transverse condition, we get the following equation for $\bar{\lambda}^{(s-1)}$ :

$$
\begin{aligned}
& {\left[\square+\frac{d+2 s-6}{d+2 s-4} u \cdot \partial \partial_{u} \cdot \partial-\frac{1}{d+2 s-4} u^{2}\left(\partial_{u} \cdot \partial\right)^{2}\right] \bar{\lambda}^{(s-1)}} \\
& +\left[\left(1-\frac{u^{2} \partial_{u}^{2}}{2(d+2 s-4)}\right) \partial_{u} \cdot \partial-\frac{u \cdot \partial \partial_{u}^{2}}{d+2 s-4}\right] \overline{\bar{h}}^{(s)}=0 .
\end{aligned}
$$


By acting a $r$-ple divergence of the above equation, we get $\left(\partial_{u} \cdot \partial\right)^{r} \bar{\lambda}^{(s-1)}$ in terms of $\left(\partial_{u} \cdot \partial\right)^{r+1} \bar{\lambda}^{(s-1)},\left(\partial_{u} \cdot \partial\right)^{r+2} \bar{\lambda}^{(s-1)}$ and $\overline{\bar{h}}^{(s)}$ :

$$
\begin{aligned}
& \left(\partial_{u} \cdot \partial\right)^{r} \bar{\lambda}^{(s-1)}= \\
& \quad \frac{1}{(r+1)(d+2 s-r-4)}\left[(d+2(s-r)-6) \frac{u \cdot \partial}{\square}\left(\partial_{u} \cdot \partial\right)^{r+1} \bar{\lambda}^{(s-1)}-\frac{u^{2}}{\square}\left(\partial_{u} \cdot \partial\right)^{r+2} \bar{\lambda}^{(s-1)}\right] \\
& \quad+\frac{d+2 s-4}{(r+1)(d+2 s-r-4)} \frac{\left(\partial_{u} \cdot \partial\right)^{r}}{\square}\left[\left(1-\frac{u^{2} \partial_{u}^{2}}{2(d+2 s-4)}\right) \partial_{u} \cdot \partial-\frac{u \cdot \partial \partial_{u}^{2}}{d+2 s-4}\right] \overline{\bar{h}}^{(s)} .
\end{aligned}
$$

Thus, one can solve iteratively the multiple divergences of $\bar{\lambda}^{(s-1)}$, and finally $\bar{\lambda}^{(s-1)}$ itself. This procedure is unique, and finally the gauge parameters $\lambda^{(s-1)}$ and $\sigma^{(s-2)}$ are uniquely determined by the traceless and transverse gauge conditions on $h^{(s)}$.

Second method: In fact it is possible to find a closed expression of $h_{\mathrm{TT}}^{(s)}$. First, we project $h^{(s)}$ on its transverse part $h_{\mathrm{T}}^{(s)}$ as

$$
h_{\mathrm{T}}^{(s)}(x, u)=\frac{1}{s !}\left(u \cdot \partial_{w}-\frac{u \cdot \partial \partial_{w} \cdot \partial}{\square}\right)^{s} h^{(s)}(x, w)=\left(\frac{\partial_{\tilde{u}} \cdot \partial}{\square}\right)^{s} \mathscr{R}^{(s)}(u, \tilde{u}),
$$

where $\mathscr{R}^{(s)}$ is the deWit-Freedman curvature [58]:

$$
\mathscr{R}^{(s)}(u, \tilde{u})=\frac{1}{s !}\left(u \cdot \partial_{w} \tilde{u} \cdot \partial-u \cdot \partial \tilde{u} \cdot \partial_{w}\right)^{s} h^{(s)}(x, w) .
$$

It is also possible to express $h_{\mathrm{T}}^{(s)}$ as

$$
h_{\mathrm{T}}^{(s)}(x, u)=\sum_{n=0}^{s} \frac{(-1)^{n}}{n !} \frac{(u \cdot \partial)^{n}\left(\partial_{u} \cdot \partial\right)^{n}}{\square^{n}} h^{(s)}(x, u)=: e^{-\frac{u \cdot \partial \partial_{u} \cdot \partial}{\square}}: h^{(s)}(x, u),
$$

where :: is the normal product. The projection to $h_{\mathrm{TT}}^{(s)}$ can be obtained by using the identity:

$$
\left[u^{2}-\frac{(u \cdot \partial)^{2}}{\square}, \partial_{u} \cdot \partial\right]=0
$$

and by taking successive traces of $h_{\mathrm{T}}^{(s)}$ as

$$
h_{\mathrm{TT}}^{(s)}(x, u)=\sum_{n} a_{n}\left(u^{2}-\frac{(u \cdot \partial)^{2}}{\square}\right)^{n}\left(\partial_{u}^{2}\right)^{n} h_{\mathrm{T}}^{(s)}(x, u) .
$$

Finally, by requiring the traceless condition, the constants $a_{n}$ are determined as

$$
a_{n}=\frac{1}{4^{n} n !\left(-\frac{d+2 s-5}{2}\right)_{n}}
$$

and replacing $h_{\mathrm{T}}^{(s)}$ by its expression and after some algebra, we get

$$
\begin{aligned}
h_{\mathrm{TT}}^{(s)}(x, u)=\sum_{n=0}^{\infty} & \frac{1}{4^{n} n !(s-2 n) !\left(\frac{1}{2}-\nu\right)_{n}}\left(u^{2}-\frac{(u \cdot \partial)^{2}}{\square}\right)^{n} \times \\
& \times\left(\partial_{w}^{2}-\frac{\left(\partial_{w} \cdot \partial\right)^{2}}{\square}\right)^{n}\left(u \cdot \partial_{w}-\frac{u \cdot \partial \partial_{w} \cdot \partial}{\square}\right)^{s-2 n} h^{(s)}(x, w) .
\end{aligned}
$$


The projector $P_{\mathrm{TT}}^{(s)}$ can be deduced as

$$
\mathcal{P}_{\mathrm{TT}}^{(s)}=\sum_{n=0}^{[s / 2]} \frac{1}{4^{n} n !\left(-\frac{d+2 s-5}{2}\right)_{n}}\left(u^{2}-\frac{(u \cdot \partial)^{2}}{\square}\right)^{n}: e^{-\frac{u \cdot \partial \partial_{u} \cdot \partial}{\square}}:\left(\partial_{u}^{2}-\frac{\left(\partial_{u} \cdot \partial\right)^{2}}{\square}\right)^{n} .
$$

Notice that $\square^{s} \mathcal{P}_{\mathrm{TT}}^{(s)}$ is a local operator.

Finally, the uniqueness of the gauge-fixing procedure under (F.1) enables us to easily obtain the contraction of the spin $s$ Weyl tensors $\mathscr{C}^{(s)}{ }^{8}$ Since the latter is invariant under (F.1), we first gauge fix $h^{(s)}$ to $h_{\mathrm{TT}}^{(s)}$. Then, taking into account that $\mathscr{C}^{(s)}$ contains $s$ derivatives acting on $h_{\mathrm{TT}}^{(s)}$, there exists only one possible expression for $\left\langle\mathscr{C}^{(s)} \mid \mathscr{C}^{(s)}\right\rangle$ expressed in terms of $h_{\mathrm{TT}}^{(s)}$ which is $\left\langle\left\langle h_{\mathrm{TT}}^{(s)} \mid(-\square)^{s} h_{\mathrm{TT}}^{(s)}\right\rangle\right.$ up to an overall constant.

Open Access. This article is distributed under the terms of the Creative Commons Attribution License which permits any use, distribution and reproduction in any medium, provided the original author(s) and source are credited.

\section{References}

[1] R. Argurio, G. Barnich, G. Bonelli and M. Grigoriev eds., Higher-Spin Gauge Theories, in proceedings of First Solvay Workshop, Brussels, 12-14 May 2004, International Solvay Institutes for Physics and Chemistry (2006)

[http://www.ulb.ac.be/sciences/ptm/pmif/Solvay1proc.pdf].

[2] M. Bianchi and V. Didenko, Massive higher spin multiplets and holography, hep-th/0502220 [INSPIRE].

[3] D. Francia and C. Hull, Higher-spin gauge fields and duality, hep-th/0501236 [INSPIRE].

[4] N. Bouatta, G. Compere and A. Sagnotti, An Introduction to free higher-spin fields, hep-th/0409068 [INSPIRE].

[5] X. Bekaert, S. Cnockaert, C. Iazeolla and M. Vasiliev, Nonlinear higher spin theories in various dimensions, hep-th/0503128 [INSPIRE].

[6] A. Sagnotti, E. Sezgin and P. Sundell, On higher spins with a strong $S p(2, R)$ condition, hep-th/0501156 [INSPIRE].

[7] D. Sorokin, Introduction to the classical theory of higher spins, AIP Conf. Proc. 767 (2005) 172 [hep-th/0405069] [INSPIRE].

[8] D. Francia and A. Sagnotti, Higher-spin geometry and string theory, J. Phys. Conf. Ser. 33 (2006) 57 [hep-th/0601199] [inSPIRE].

[9] X. Bekaert, N. Boulanger and P. Sundell, How higher-spin gravity surpasses the spin two barrier: no-go theorems versus yes-go examples, arXiv:1007.0435 [INSPIRE].

[10] A. Sagnotti, Notes on Strings and Higher Spins, arXiv:1112.4285 [InSPIRE].

[11] G. Bonelli, On the covariant quantization of tensionless bosonic strings in AdS space-time, JHEP 11 (2003) 028 [hep-th/0309222] [INSPIRE].

[12] G. Bonelli, On the boundary gauge dual of closed tensionless free strings in AdS, JHEP 11 (2004) 059 [hep-th/0407144] [INSPIRE].

\footnotetext{
${ }^{8}$ The spin $s$ Weyl tensor is the mixed symmetry traceless projection of the curvature $\mathscr{R}^{(s)}(u, \tilde{u})$
} 
[13] D. Polyakov, Interactions of Massless Higher Spin Fields From String Theory, Phys. Rev. D 82 (2010) 066005 [arXiv:0910.5338] [inSPIRE].

[14] D. Polyakov, Gravitational Couplings of Higher Spins from String Theory, Int. J. Mod. Phys. A 25 (2010) 4623 [arXiv:1005.5512] [inSPIRE].

[15] D. Polyakov, Higher Spins and Open Strings: Quartic Interactions, Phys. Rev. D 83 (2011) 046005 [arXiv:1011.0353] [InSPIRE].

[16] D. Polyakov, A String Model for AdS Gravity and Higher Spins, Phys. Rev. D 84 (2011) 126004 [arXiv:1106.1558] [InSPIRE].

[17] A. Sagnotti and M. Taronna, String Lessons for Higher-Spin Interactions, Nucl. Phys. B 842 (2011) 299 [arXiv: 1006.5242] [INSPIRE].

[18] B. Sundborg, Stringy gravity, interacting tensionless strings and massless higher spins, Nucl. Phys. Proc. Suppl. 102 (2001) 113 [hep-th/0103247] [INSPIRE].

[19] E. Witten, Spacetime Reconstruction, talk given at J.H. Schwarz $60^{\text {th }}$ Birthday Conference, Cal Tech, 2-3 November 2001 [http://theory.caltech.edu/jhs60/witten/1.html].

[20] A. Mikhailov, Notes on higher spin symmetries, hep-th/0201019 [INSPIRE].

[21] A.A. Tseytlin, On limits of superstring in $A d S_{5} \times S^{5}$, Theor. Math. Phys. 133 (2002) 1376 [hep-th/0201112] [INSPIRE].

[22] E. Sezgin and P. Sundell, Massless higher spins and holography, Nucl. Phys. B 644 (2002) 303 [Erratum ibid. B 660 (2003) 403] [hep-th/0205131] [INSPIRE].

[23] A.Y. Segal, Conformal higher spin theory, Nucl. Phys. B 664 (2003) 59 [hep-th/0207212] [INSPIRE].

[24] I. Klebanov and A. Polyakov, AdS dual of the critical $O(N)$ vector model, Phys. Lett. B 550 (2002) 213 [hep-th/0210114] [INSPIRE].

[25] J. Maldacena and A. Zhiboedov, Constraining Conformal Field Theories with A Higher Spin Symmetry, arXiv:1112.1016 [INSPIRE].

[26] E. Witten, Anti-de Sitter space and holography, Adv. Theor. Math. Phys. 2 (1998) 253 [hep-th/9802150] [INSPIRE].

[27] M. Vasiliev, Higher spin gauge theories in any dimension, Comptes Rendus Physique 5 (2004) 1101 [hep-th/0409260] [INSPIRE].

[28] M. Vasiliev, Higher spin gauge theories in various dimensions, Fortsch. Phys. 52 (2004) 702 [hep-th/0401177] [INSPIRE].

[29] L. Girardello, M. Porrati and A. Zaffaroni, 3-D interacting CFTs and generalized Higgs phenomenon in higher spin theories on AdS, Phys. Lett. B 561 (2003) 289 [hep-th/0212181] [INSPIRE].

[30] E. Sezgin and P. Sundell, Holography in $4 D$ (super) higher spin theories and a test via cubic scalar couplings, JHEP 07 (2005) 044 [hep-th/0305040] [INSPIRE].

[31] A.C. Petkou, Evaluating the AdS dual of the critical $O(N)$ vector model, JHEP 03 (2003) 049 [hep-th/0302063] [INSPIRE].

[32] A.C. Petkou, Holography, duality and higher-spin theories, hep-th/0410116 [INSPIRE]. 
[33] R. Manvelyan and W. Rühl, The Masses of gauge fields in higher spin field theory on the bulk of $A d S_{4}$, Phys. Lett. B 613 (2005) 197 [hep-th/0412252] [INSPIRE].

[34] R. Manvelyan and W. Rühl, The Off-shell behaviour of propagators and the Goldstone field in higher spin gauge theory on $A d S_{d+1}$ space, Nucl. Phys. B 717 (2005) 3 [hep-th/0502123] [INSPIRE].

[35] R. Manvelyan and W. Rühl, The Quantum one loop trace anomaly of the higher spin conformal conserved currents in the bulk of AdS $S_{4}$, Nucl. Phys. B 733 (2006) 104 [hep-th/0506185] [INSPIRE].

[36] R. Manvelyan and W. Rühl, The Structure of the trace anomaly of higher spin conformal currents in the bulk of $A d S_{4}$, Nucl. Phys. B 751 (2006) 285 [hep-th/0602067] [INSPIRE].

[37] R. Manvelyan, K. Mkrtchyan and W. Rühl, Ultraviolet behaviour of higher spin gauge field propagators and one loop mass renormalization, Nucl. Phys. B 803 (2008) 405 [arXiv:0804.1211] [INSPIRE].

[38] R. Metsaev, Shadows, currents and AdS, Phys. Rev. D 78 (2008) 106010 [arXiv:0805.3472] [INSPIRE].

[39] R. Metsaev, Gauge invariant two-point vertices of shadow fields, AdS/CFT and conformal fields, Phys. Rev. D 81 (2010) 106002 [arXiv:0907.4678] [INSPIRE].

[40] S. Giombi and X. Yin, Higher Spin Gauge Theory and Holography: The Three-Point Functions, JHEP 09 (2010) 115 [arXiv:0912.3462] [INSPIRE].

[41] S. Giombi and X. Yin, Higher Spins in AdS and Twistorial Holography, JHEP 04 (2011) 086 [arXiv: 1004.3736] [INSPIRE].

[42] A. Campoleoni, S. Fredenhagen, S. Pfenninger and S. Theisen, Asymptotic symmetries of three-dimensional gravity coupled to higher-spin fields, JHEP 11 (2010) 007 [arXiv: 1008.4744] [INSPIRE].

[43] M. Henneaux and S.-J. Rey, Nonlinear $W_{\text {infinity }}$ as Asymptotic Symmetry of Three-Dimensional Higher Spin Anti-de Sitter Gravity, JHEP 12 (2010) 007 [arXiv: 1008.4579] [INSPIRE].

[44] C. Fronsdal, Singletons and Massless, Integral Spin Fields on de Sitter Space (Elementary Particles in a Curved Space. 7., Phys. Rev. D 20 (1979) 848 [InSPIRE].

[45] X. Bekaert, E. Joung and J. Mourad, Effective action in a higher-spin background, JHEP 02 (2011) 048 [arXiv: 1012.2103] [INSPIRE].

[46] J.W. York, Role of conformal three geometry in the dynamics of gravitation, Phys. Rev. Lett. 28 (1972) 1082 [INSPIRE].

[47] G. Gibbons and S. Hawking, Action Integrals and Partition Functions in Quantum Gravity, Phys. Rev. D 15 (1977) 2752 [InSPIRE].

[48] M. Henningson and K. Skenderis, The Holographic Weyl anomaly, JHEP 07 (1998) 023 [hep-th/9806087] [INSPIRE].

[49] E. Fradkin and A.A. Tseytlin, Conformal supergravity, Phys. Rept. 119 (1985) 233 [InSPIRE].

[50] E. Fradkin and V.Y. Linetsky, Cubic interaction in conformal theory of integer higher spin fields in four-dimensional space-time, Phys. Lett. B 231 (1989) 97 [INSPIRE].

[51] E. Fradkin and V.Y. Linetsky, Conformally invariant field theory with higher spins, Sov. Phys. Dokl. 35 (1990) 256 [InSPIRE]. 
[52] D. Francia and A. Sagnotti, Minimal local Lagrangians for higher-spin geometry, Phys. Lett. B 624 (2005) 93 [hep-th/0507144] [INSPIRE].

[53] D. Francia, J. Mourad and A. Sagnotti, Current Exchanges and Unconstrained Higher Spins, Nucl. Phys. B 773 (2007) 203 [hep-th/0701163] [inSPIRE].

[54] D. Francia, J. Mourad and A. Sagnotti, (A)dS exchanges and partially-massless higher spins, Nucl. Phys. B 804 (2008) 383 [arXiv:0803.3832] [inSPIRE].

[55] M. Vasiliev, Cubic Vertices for Symmetric Higher-Spin Gauge Fields in (A)d $S_{d}$, Nucl. Phys. B 862 (2012) 341 [arXiv:1108.5921] [INSPIRE].

[56] E. Joung and M. Taronna, Cubic interactions of massless higher spins in (A)dS: metric-like approach, Nucl. Phys. B 861 (2012) 145 [arXiv:1110.5918] [INSPIRE].

[57] X. Bekaert, E. Joung and J. Mourad, On higher spin interactions with matter, JHEP 05 (2009) 126 [arXiv:0903.3338] [INSPIRE].

[58] B. de Wit and D.Z. Freedman, Systematics of Higher Spin Gauge Fields, Phys. Rev. D 21 (1980) 358 [INSPIRE]. 